\title{
Synthesis of $( \pm)$-Pisonivanone and Other Analogs as Potent Antituberculosis Agents
}

\author{
A. Vasu Babu, ${ }^{1}$ A. Rambabu, ${ }^{1}$ P. V. Giriprasad, ${ }^{2}$ R. Surya Chandra Rao, ${ }^{1}$ and B. Hari Babu ${ }^{1}$ \\ ${ }^{1}$ Department of Chemistry, Acharya Nagarjuna University, Nagarjunanagar, Andhrapadesh, Guntur 522510, India \\ ${ }^{2}$ Department of Microbiology, Sri Venkateswara University, Andhrapadesh, Tirupati 517502, India
}

Correspondence should be addressed to B. Hari Babu; dr.b.haribabu@gmail.com

Received 29 June 2012; Accepted 16 September 2012

Academic Editor: Shino Homma-Takeda

Copyright (C) 2013 A.Vasu Babu et al. This is an open access article distributed under the Creative Commons Attribution License, which permits unrestricted use, distribution, and reproduction in any medium, provided the original work is properly cited.

\begin{abstract}
A new class of alkylated chalcones and flavanones was synthesised and screened for antituberculosis, antixoidant, and cytotoxic activities. The desired compounds were synthesised using methyl substituted 2-hydroxyacetophenone as a key intermediate. The acetophenone derivative having methyl substitution was prepared in turn from methtylated phloroglucinol by formylation (by Vilsmeier-Haack reaction), followed by reduction with Wolf-Kishnner approach, and finally acetylation was involved. Among 17 compounds, compound $\mathbf{5}$ and compound $\mathbf{4 a}$ inhibited M. tuberculosis at minimum inhibitory concentration (MIC) in the range between $25 \mu \mathrm{g} / \mathrm{mL}$ and $50 \mu \mathrm{g} / \mathrm{mL}$. The remaining other 15 compounds also potently inhibited M. tuberculosis at MIC in range between $50 \mu \mathrm{g} / \mathrm{mL}$ and $100 \mu \mathrm{g} / \mathrm{mL}$. Some of these compounds also showed moderate antioxidant and cytotoxic activities.
\end{abstract}

\section{Introduction}

A survey of the literature revealed that many flavonoids benefit human health [1]. Among these large class of compounds some of the alkylated flavonoids isolated from plants also act as antimalarial [2], antioxidant [3], anticancer [46], anti-inflammatory [7], and antimicrobial agents [8] like unsubstituted flavonoids. Recently antitubercular chromones and flavonoids [9] having methyl substitution were isolated from Pisonia aculeate, which belongs to Nyctaginaceae family. Among these the titled compound pisonivanone was reported as a potent antituberculosis compound (MIC = $12.5 \mu \mathrm{g} / \mathrm{mL}$ ). So in order to develop the activity profile of the methyl substituted flavonoids, an attempt was made to synthesize pisonivanone for the first time and other new chalcones and flavanones. These synthesized compounds were screened for some biological activities like antioxidant, cytotoxic, and antituberculosis. The scheme for the synthesis of Pisonivanone and other analogs was given below (Scheme 1).

2-Hydroxy-3-methyl-4,6-dimethoxy acetophenone (1) was synthesised in different ways [10]. In the present work, we aimed to synthesise the key intermediate acetophenone (1) from phloroglucinol using regular conventional methods like methylation [11], formylation [12], Wolf-Kishner reduction, and acetylation [12] with better yields (Scheme 2). All the intermediates were confirmed by comparing the spectral data and melting points with the literature.

Chalcones are versatile molecules and intermediates for synthesis of different heterocyclic compounds [13]. Chalcones have been synthesised in different ways [14, 15]. The chalcones (3a-h) were synthesised by Claisen-Schmidt reaction. In this reaction, condensation was between acetophenone (1) and substituted aromatic aldehydes (2a-h) in the presence of aqueous $\mathrm{KOH}$ and ethanol at room temperature.

Flavanones (Compound $\mathbf{4 a - h}$ ) were prepared on cyclisation of chalcones (Compounds $\mathbf{3} \mathbf{a}-\mathbf{h}$, resp.) in the presence of alcoholic $\mathrm{H}_{2} \mathrm{SO}_{4}$. Initially, chalcones were dissolved in alcoholic $\mathrm{H}_{2} \mathrm{SO}_{4}$ and refluxed at $60^{\circ} \mathrm{C}$ for $24 \mathrm{hrs}$. After cooling, alcohol was removed under vacuum. When ice cold water added to the reaction, mass pale reddish colour solids were separated. These solids were extracted with ethyl acetate. This ethyl acetate layer dried over sodium sulphate. This ethyl 


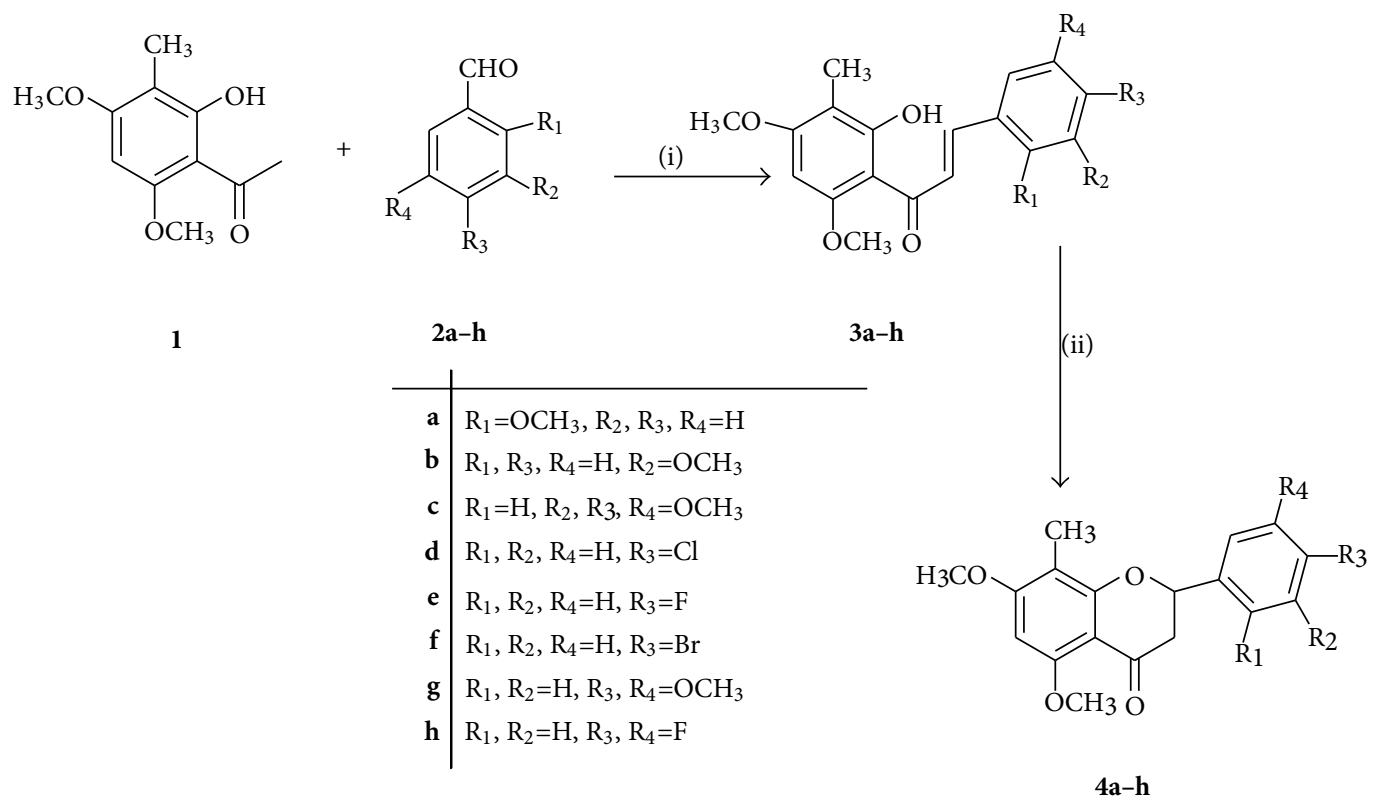

Scheme 1: Reagents and conditions: (i) $\mathrm{KOH} / \mathrm{EtOH}, 24 \mathrm{~h}$. (ii) EtOH, $\mathrm{H}_{2} \mathrm{SO}_{4}, 60^{\circ} \mathrm{C} .24 \mathrm{~h}$.<smiles>COc1cc(OC)cc(OC)c1</smiles>

1

Scheme 2: Reagents and conditions: (i) DMS/Acetone, $\mathrm{K}_{2} \mathrm{CO}_{3}, 70^{\circ} \mathrm{C} / 6 \mathrm{~h}$, (ii) DMF/POCl $3,0^{\circ} \mathrm{C} / 1 \mathrm{~h}$, (iii) $\mathrm{NH}_{2} \mathrm{NH}_{2} \cdot \mathrm{H}_{2} \mathrm{O} / \mathrm{KOH}$, ethylene glycol, $80-90^{\circ} \mathrm{C}$ for $2 \mathrm{~h}$ followed by heating at 130 to $140^{\circ} \mathrm{C}$ for $2 \mathrm{~h}$, (iv) $\mathrm{CH}_{3} \mathrm{COCl} / \mathrm{AlCl}_{3}, \mathrm{MDC} .0^{\circ} \mathrm{C} / 2 \mathrm{~h}$.

acetate layer was concentrated and washed with diethyl ether to remove adherent chalcone.

Finally, the targeted compound $\mathbf{5}$ (pisonivanone) was synthised from Compound $\mathbf{4 a}$ on demethylation. Pyridinium hydrochloride was added to Compound $\mathbf{4 a}$, at inert conditions in 1:10 ratio and refluxed for 1 hour at $200^{\circ} \mathrm{C}$. Then reaction mixture poured in ice cold water and extracted with ethyl acetate. This ethyl acetate layer dried over sodium sulphate and on column chromatography with ethyl acetate, and hexane pure pisonivanone was separated (yellow crystalline solid, yield 55\%) (see Scheme 3).

\section{Discussions}

Based on the literature, all these synthesised Compounds 3a-h, 4a-h, Compound-5 (pisonivanone) were screened for their biological activities like antitubercular, antioxidant, and cytotoxicity. All these methyl substituted flavonoids were showed potent antitubercular activity against $M$. tuberculosis strain (H37Rv). Possibly the C-8 methyl group in basic skeleton of the molecules plays an important role in the antimycobacterial activity. Among 17 compounds Compound $\mathbf{5}$ showed minimum inhibitory concentration (MIC) at $25 \mu \mathrm{g} / \mathrm{mL}$ and Compound $\mathbf{4 a}$ inhibited M. tuberculosis at minimum inhibitory concentration (MIC) $\geq 50 \mu \mathrm{g} / \mathrm{mL}$. The remaining other 15 compounds also potently inhibited M. tuberculosis at MIC in range between $50 \mu \mathrm{g} / \mathrm{mL}$ and $100 \mu \mathrm{g} / \mathrm{mL}$. These results suggest that the methyl substituted flavonoids may consider as potent antituberculosis agents, and there is a scope to improve the activity further by preparing different possible analogs. The results given in Tables 2 and 3 for chalcones and flavanones showed the compounds have moderate antioxidant and cytotoxic activities.

\section{Experimental}

3.1. Materials and Methods. Phloroglucinol and other chemicals used were of AR grade (Merck). Melting points were taken in open glass capillary by using Remi melting point apparatus and were uncorrected. ${ }^{1} \mathrm{H}$ NMR spectra were recorded on Brucker $400 \mathrm{MHz}$ and ${ }^{13} \mathrm{C} \mathrm{NMR}$ was on Brucker $100 \mathrm{MHz}$ spectrophotometers. Mass spectra recorded on LCMS Agilent LC-1100 series.

3.1.1. Synthesis of 2,4,6-Trimethoxy Phloroglucinol. A solution of anhydrous phloroglucinol ( $50 \mathrm{~g}, 0.396 \mathrm{~mole})$ in 
<smiles>COc1ccccc1C1CC(=O)c2c(OC)cc(OC)c(C)c2O1</smiles>

Compound-4a

\section{$\Delta 200^{\circ} \mathrm{C} / 1 \mathrm{~h}$ \\ Pyridinium hydrochloride}<smiles>Cc1c(O)cc(O)c2c1OC(c1ccccc1O)CC2=O</smiles>

( \pm )-pisonivanone Compound-5

SCHEME 3

dry acetone $(700 \mathrm{~mL})$ is refluxed for $6 \mathrm{~h}$ at $60^{\circ} \mathrm{C}$ under anhydrous conditions with dimethyl sulphate $(112.10 \mathrm{~mL}$, $148 \mathrm{~g}, 1.17 \mathrm{~mole})$ in presence of potassium carbonate $(280 \mathrm{~g}$, 2.02 mole). The acetone layer was filtered and distilled. The residue on column chromatography with hexane and ethyl acetate $(95: 5)$ trimethoxy phloroglucinol separated. (\% of yield 94 , white solid, M.P- $52-54^{\circ} \mathrm{C}$.)

3.1.2. Synthesis of 2,4,6-Trimethoxy Benzaldehyde. Dimethyl formamide $(36.8 \mathrm{~mL}, 1.3 \mathrm{eq}, 0.4797$ mole) is added to trimethoxy phloroglucinol (62 g, 0.369 mole, 1 eq) and cooled $\left(0^{\circ} \mathrm{C}\right.$.) Phosphorous oxychloride $(153.33 \mathrm{~mL}$, $65.63 \mathrm{gm}, 1.16 \mathrm{eq})$ is added drop wise to this cooling mixture slowly under anhydrous conditions. The mixture is allowed to stand for $1 \mathrm{~h}$ at room temperature. The reaction mixture poured into crushed ice and basified with $8 \mathrm{~N} \mathrm{KOH}$. The filtered solid dissolved in ethyl acetate and treated with brine. The ethyl acetate layer dried over sodium sulphate and concentrated. (\% of yield-91.6, yellow viscous solid, M.P- $119-120^{\circ}$ C.)

3.1.3. Synthesis of 3-Methyl-2,4,6 Trimethoxy Phloroglucinol. 2,4,6-Trimethoxy benzaldehyde is dissolved in ethylene gly$\mathrm{col}$ and hydrazine hydrate is added. Powdered $\mathrm{KOH}$ portion wise added to the reaction mixture and heated to $80-90^{\circ} \mathrm{C}$ for $2 \mathrm{~h}$ then foaming is formed. The reaction mixture heated to $135-145^{\circ} \mathrm{C}$ for another $2 \mathrm{~h}$. Then the reaction mixture is poured into ice cold water, acidified with dil $\mathrm{HCl}$, and extracted with hexane. Hexane layer is washed with sodium carbonate, water, and brine. This hexane layer dried over sodium sulphate and concentrated. (\% of yield 80 , colour less liquid.)

3.1.4. Synthesis of 2-Hydroxy-3-methyl-4,6-dimethoxy Acetophenone (1). A solution of 3-methyl-2,4,6 Trimethoxy phloroglucinol (55 g, 0.302 mole) in absolute ether $(450 \mathrm{~mL})$ is added to a cold solution of anhydrous aluminum chloride (86.39 g, 0.64 mole, $2.2 \mathrm{eq})$ in absolute ether $(100 \mathrm{~mL})$. The cooled $\left(0-5^{\circ} \mathrm{C}\right)$ and stirred solution is treated with acetyl chloride (25.21 mL, 0.319 mole, $1.2 \mathrm{eq}$ ), added dropwise during $1 \mathrm{hr}$. The mixture is stirred for $4 \mathrm{hr}$ more. The whole reaction is done under anhydrous condition. The mixture is left overnight at room temperature and treated in cold with crushed ice. It is acidified with $5 \mathrm{~N} \mathrm{HCl}$ and extracted with ethyl acetate. This ethyl acetate layer dried over sodium sulphate and concentrated. (\% of yield 85, light green color crystals, M.P-90-92 ${ }^{\circ}$ C.)

3.1.5. General Method for Synthesis of Chalcones (Compound $3 \mathbf{a}-\mathbf{h})$. To a solution of acetophenone $(0.5 \mathrm{~g}, 0.00238 \mathrm{~mole})$ and substituted benzaldehydes (2a-h) (1 eq) in ethanol $(10 \mathrm{~mL})$ is added aqueous potassium hydroxide $(0.599 \mathrm{~g}$, $4.5 \mathrm{eq}$ in $0.5 \mathrm{~mL}$ water). The mixture is stirred at room temperature for $24 \mathrm{~h}$. To this ice cold water added and separated solid was filtered. The separated solid was dissolved in ethyl acetate, died over sodium sulphate, and concentrated under vacuum.

3.1.6. Spectral Data of Synthesized Compounds. Compound 3a. It is having the molecular formula $\mathrm{C}_{19} \mathrm{H}_{20} \mathrm{O}_{5}$ which showed $(\mathrm{M}+\mathrm{H})^{+}$at 329 in LC-MS. So the molar mass of the Compound 3a is $328 \mathrm{~g} / \mathrm{mol}$. Yield $-85 \%$, M.P $-157-160^{\circ} \mathrm{C}$.

${ }^{1} \mathrm{H} \mathrm{NMR}\left(\mathrm{CDCl}_{3}, 400 \mathrm{MHz}, \delta\right.$ in ppm): $3 \mathrm{H}, \mathrm{s}, 3.991$ (Ar$\left.\mathrm{OCH}_{3}\right), 3 \mathrm{H}, \mathrm{s} 3.932\left(\mathrm{Ar}-\mathrm{OCH}_{3}\right), 3 \mathrm{H}, \mathrm{s}, 3.917\left(\mathrm{Ar}-\mathrm{OCH}_{3}\right), 1 \mathrm{H}$, s, $14.045(\mathrm{Ar}-\mathrm{OH}), 2 \mathrm{H}, \mathrm{d}, 7.98\left(\mathrm{Ar}^{1}-\mathrm{H}\right), 1 \mathrm{H}, \mathrm{dd}, 7.71\left(\mathrm{Ar}^{1}-\mathrm{H}\right)$, $1 \mathrm{H} \mathrm{m} 7.4\left(\mathrm{Ar}^{1}-\mathrm{H}\right), 1 \mathrm{H}, \mathrm{d}, 7.05\left(\mathrm{Ar}^{1}-\mathrm{H}\right), 1 \mathrm{H}, \mathrm{d}, 7.1(\mathrm{C}=\mathrm{C}-\mathrm{H})$, $1 \mathrm{H}, \mathrm{d}, 6.3(\mathrm{C}=\mathrm{C}-\mathrm{H})$.

${ }^{13} \mathrm{C} \mathrm{NMR}\left(\mathrm{CDCl}_{3}, 100 \mathrm{MHz}, \delta\right.$ in ppm): $192.72(\mathrm{C}=\mathrm{O})$, $7.32\left(\mathrm{ArCH}_{3}\right), 56.1\left(\mathrm{Ar}-\mathrm{OCH}_{3}\right), 55.8\left(\mathrm{Ar}-\mathrm{OCH}_{3}\right), 55.69\left(\mathrm{Ar}^{1}-\right.$ $\left.\mathrm{OCH}_{3}\right), 163.6,162.9,161.5$ (Ar-C-O), 158.32 ( $\left.\mathrm{Ar}^{1}-\mathrm{C}\right), 87.65$ (Ar-C), 105.6, 104.2 (Ar-C), 120.87, 111.88 (C=C), 137.28, $131.93,128.96,127.77,123.30\left(\mathrm{Ar}^{1}-\mathrm{C}\right)$.

Compound 3b. It is having the molecular formula $\mathrm{C}_{19} \mathrm{H}_{20} \mathrm{O}_{5}$ which showed $(\mathrm{M}+\mathrm{H})^{+}$at 329 in LC-MS. So the molar mass of the compound $\mathbf{3 b}$ is $328 \mathrm{~g} / \mathrm{mol}$. Yield $-80 \%$, M.P- $125-130^{\circ} \mathrm{C}$.

${ }^{1} \mathrm{H}$ NMR $\left(\mathrm{CDCl}_{3}, 400 \mathrm{MHz}, \delta\right.$ in ppm): 1H, d, 14.03 (Ar$\mathrm{OH}), 1 \mathrm{H}, \mathrm{d}, 7.8\left(\mathrm{Ar}^{1}-\mathrm{H}\right), 1 \mathrm{H}, \mathrm{d}, 7.7\left(\mathrm{Ar}^{1}-\mathrm{H}\right), 1 \mathrm{H}, \mathrm{s}, 5.99(\mathrm{Ar}-$ $\mathrm{H}), 1 \mathrm{H}, \mathrm{s}, 7.29\left(\mathrm{Ar}^{1}-\mathrm{H}\right), 1 \mathrm{H}, \mathrm{m}, 7.33\left(\mathrm{Ar}^{1}-\mathrm{H}\right), 1 \mathrm{H}, \mathrm{d}, 7.21$ $(\mathrm{C}=\mathrm{C}-\mathrm{H}) 1 \mathrm{H}, \mathrm{d} 6.9(\mathrm{C}=\mathrm{C}-\mathrm{H}), 3 \mathrm{H}, \mathrm{s}, 3.9\left(\mathrm{Ar}^{1} \mathrm{OCH}_{3}\right), 3 \mathrm{H}, \mathrm{s}$, $3.9\left(\mathrm{Ar}-\mathrm{OCH}_{3}\right), 3 \mathrm{H}, \mathrm{s}, 3.8\left(\mathrm{Ar}-\mathrm{OCH}_{3}\right), 3 \mathrm{H}, \mathrm{s}, 2.03\left(\mathrm{Ar}-\mathrm{CH}_{3}\right)$.

${ }^{13} \mathrm{C} \mathrm{NMR}\left(\mathrm{CDCl}_{3}, 100 \mathrm{MHz}, \delta\right.$ in ppm): $193.00(\mathrm{O}=\mathrm{C})$, 164.27, $163.65\left(\mathrm{Ar}-\mathrm{C}-\mathrm{OCH}_{3}\right), 161.13(\mathrm{Ar}-\mathrm{OH}), 159.93\left(\mathrm{Ar}^{1}-\right.$ $\left.\mathrm{OCH}_{3}\right), 141.72(\mathrm{C}=\mathrm{C}-\mathrm{Ar}), 128.35(\mathrm{C}=\mathrm{C}-\mathrm{C}=\mathrm{O}), 86.43(\mathrm{Ar}-$ C), $106.40\left(\mathrm{Ar}-\mathrm{C}-\mathrm{CH}_{3}\right), 106.22(\mathrm{Ar}-\mathrm{C}-\mathrm{C}=\mathrm{O}), 137.1,129.80$, 120.87, 115.51, $113.63\left(\mathrm{Ar}^{1}-\mathrm{C}\right), 55.84,55.49\left(\mathrm{Ar}-\mathrm{OCH}_{3}\right)$, $55.28\left(\mathrm{Ar}^{1}-\mathrm{OCH}_{3}\right), 7.23\left(\mathrm{Ar}-\mathrm{CH}_{3}\right)$. 
Compound 3c. Compound having the molecular formula $\mathrm{C}_{21} \mathrm{H}_{24} \mathrm{O}_{7}$ is showed molecular ion peak at $389.2(\mathrm{Neg})$ in LC-MS. Molecular weight of the compound $3 \mathrm{c}$ is $388 \mathrm{~g} / \mathrm{mol}$. Yield-86\%, M.P-200-205 ${ }^{\circ}$ C.

${ }^{1} \mathrm{H} \mathrm{NMR}\left(\mathrm{CDCl}_{3}, 400 \mathrm{MHz}, \delta\right.$ in ppm): $1 \mathrm{H}, \mathrm{s}, 14.09$ (Ar$\mathrm{OH}), 3 \mathrm{H}, \mathrm{s} 3.93\left(\mathrm{Ar}^{1}-\mathrm{OCH}_{3}\right), 6 \mathrm{H}, \mathrm{s} 3.9\left(\mathrm{Ar}^{1}-\mathrm{OCH}_{3}\right), 3 \mathrm{H}, \mathrm{s} 3.9$ $\left(\mathrm{Ar}-\mathrm{OCH}_{3}\right), 3 \mathrm{H}, \mathrm{s} 3.8\left(\mathrm{Ar}-\mathrm{OCH}_{3}\right), 3 \mathrm{H} 2.03\left(\mathrm{Ar}-\mathrm{CH}_{3}\right), 1 \mathrm{H}, \mathrm{s}$ 5.99 (Ar-H), 2H, s $6.83\left(\mathrm{Ar}^{1}-\mathrm{H}\right), 1 \mathrm{H} \mathrm{d} 7.7(\mathrm{C}=\mathrm{C}-\mathrm{H}), 1 \mathrm{H}, \mathrm{d}$ $7.69(\mathrm{C}=\mathrm{C}-\mathrm{H})$.

${ }^{13} \mathrm{C} \mathrm{NMR}\left(\mathrm{CDCl}_{3}, 100 \mathrm{~Hz}, \delta\right.$ in ppm): $192.75(\mathrm{O}=\mathrm{C})$, 164.29, 163.57, (Ar-C-OCH $\left.{ }_{3}\right), 161.02(\mathrm{Ar}-\mathrm{OH}), 153.44\left(\mathrm{Ar}^{1}-\right.$ $\left.\mathrm{OCH}_{3}\right), 141.97\left(\mathrm{Ar}^{1}-\mathrm{OCH}_{3}\right), 141.97\left(\mathrm{Ar}^{1}-\mathrm{OCH}_{3}\right), 140.21$ $(\mathrm{C}=\mathrm{C}-\mathrm{Ar}), 127.35(\mathrm{C}=\mathrm{C}-\mathrm{C}=\mathrm{O}), 86.43$ (Ar-C), 106.40 (Ar-C$\left.\mathrm{CH}_{3}\right), 106.24(\mathrm{Ar}-\mathrm{C}-\mathrm{C}=\mathrm{O}), 131.24,\left(\mathrm{Ar}^{1}-\mathrm{C}\right), 105.72\left(\mathrm{Ar}^{1}-\mathrm{C}\right)$, $105.72\left(\mathrm{Ar}^{1}-\mathrm{C}\right), 60.95,56.18\left(\mathrm{Ar}-\mathrm{OCH}_{3}\right), 55.79,55.4955 .28$ $\left(\mathrm{Ar}^{1}-\mathrm{OCH}_{3}\right), 7.23\left(\mathrm{Ar}-\mathrm{CH}_{3}\right)$.

Compound 3d. It is having the molecular formula $\mathrm{C}_{18} \mathrm{H}_{17} \mathrm{O}_{4} \mathrm{Cl}$ which showed molecular ion $(\mathrm{M}-\mathrm{H})^{-}$peak in negative mode at 331 in LC-MS. So the molar mass of the compound $3 \mathrm{~d}$ is $332 \mathrm{~g} / \mathrm{mol}$. Yield $-70 \%$, M.P- $185-190^{\circ} \mathrm{C}$.

${ }^{1} \mathrm{HNMR}\left(400 \mathrm{MHz}, \delta\right.$ in ppm, $\left.\mathrm{CDCl}_{3}\right): 1 \mathrm{H}, \mathrm{s}, 14.00(\mathrm{Ar}-$ $\mathrm{OH}), 3 \mathrm{H}, \mathrm{s}, 3.95\left(\mathrm{Ar}-\mathrm{OCH}_{3}\right), 3 \mathrm{H}, \mathrm{s}, 3.980\left(\mathrm{Ar}-\mathrm{OCH}_{3}\right), 3 \mathrm{H}, \mathrm{s}$, $2.037\left(\mathrm{Ar}_{-} \mathrm{CH}_{3}\right), 1 \mathrm{H}, \mathrm{s}, 5.92(\mathrm{Ar}-\mathrm{H}), 2 \mathrm{H}, \mathrm{d} 7.37\left(\mathrm{Ar}^{1}-\mathrm{H}\right), 2 \mathrm{H}, \mathrm{d}$, $7.53\left(\mathrm{Ar}^{1}-\mathrm{H}\right), 1 \mathrm{H} \mathrm{d}, 7.7(\mathrm{C}=\mathrm{C}-\mathrm{H}), 1 \mathrm{H}, \mathrm{d}, 7.8(\mathrm{C}=\mathrm{C}-\mathrm{H})$.

${ }^{13} \mathrm{CNMR}\left(100 \mathrm{MHz}, \delta\right.$ in ppm, $\left.\mathrm{CDCl}_{3}\right): 192.73(\mathrm{C}=\mathrm{O})$, 164.28, $163.76\left(\mathrm{Ar}-\mathrm{C}-\mathrm{OH}_{3}\right), 161.11$ (Ar-C-OH), 86.40 (ArC), 106.32 (Ar-C-CH $)_{3}, 106.24$ (Ar-C-C=O), 140.33 (-C=C$\mathrm{Ar}), 128.50(-\mathrm{C}=\mathrm{CH}), 135.74\left(\mathrm{Ar}^{1}-\mathrm{Cl}\right), 134.22\left(\mathrm{Ar}^{1}-\mathrm{C}\right), 129.39$ (Ar $\left.{ }^{1}-\mathrm{C}-\mathrm{H}\right), 128.50$ (Ar-C-H), 137.45(CH=C), 127.85 (C=C$\mathrm{H}) .55 .85,55.52\left(\mathrm{Ar}-\mathrm{O}-\mathrm{CH}_{3}\right), 7.24\left(\mathrm{Ar}-\mathrm{CH}_{3}\right)$.

Compound 3e. It is having the molecular formula $\mathrm{C}_{18} \mathrm{H}_{17} \mathrm{O}_{4} \mathrm{~F}$ which showed molecular ion peak in positive mode $(\mathrm{M}+\mathrm{H})^{+}$at 317 in LC-MS. So the molar mass of the compound $3 \mathrm{e}$ is $316 \mathrm{~g} / \mathrm{mol}$. Yield $-75 \%$, M.P $-165-170^{\circ} \mathrm{C}$.

${ }^{1} \mathrm{H}$ NMR $\left(400 \mathrm{MHz}, \delta\right.$ in ppm, $\left.\mathrm{CDCl}_{3}\right): 1 \mathrm{H}, \mathrm{s}, 14.01$ (Ar-OH), 1H,s, $5.985(\mathrm{Ar}-\mathrm{H}), 3 \mathrm{H}, \mathrm{s}, 3.98\left(\mathrm{Ar}-\mathrm{O}-\mathrm{CH}_{3}\right), 3 \mathrm{H}, \mathrm{s}$, $3.89\left(\mathrm{Ar}-\mathrm{O}-\mathrm{CH}_{3}\right), 3 \mathrm{H}, \mathrm{s}, 2.03\left(\mathrm{Ar}-\mathrm{CH}_{3}\right), 1 \mathrm{H}, \mathrm{d}, 8.00(\mathrm{C}=\mathrm{C}-\mathrm{H})$, 1H,d, $7.84(\mathrm{C}=\mathrm{C}-\mathrm{H}), 1 \mathrm{H}, \mathrm{m}, 7.5\left(\mathrm{Ar}^{1}-\mathrm{H}\right), 1 \mathrm{H}, \mathrm{m}, 7.3\left(\mathrm{Ar}^{1}-\mathrm{H}\right)$, 1H,m, $7.14\left(\mathrm{Ar}^{1}-\mathrm{H}\right), 1 \mathrm{H}, \mathrm{m}, 7.09\left(\mathrm{Ar}^{1}-\mathrm{H}\right)$.

${ }^{13} \mathrm{CNMR}\left(100 \mathrm{MHz}, \delta\right.$ in $\left.\mathrm{ppm}, \mathrm{CDCl}_{3}\right): 193.04(\mathrm{C}=\mathrm{O})$, $106.42\left(\mathrm{Ar}-\mathrm{C}-\mathrm{CH}_{3}\right), 164.34\left(\mathrm{Ar}-\mathrm{O}-\mathrm{CH}_{3}\right), 86.44$ (Ar-C-H), $163.80\left(\mathrm{Ar}-\mathrm{O}-\mathrm{CH}_{3}\right), 162.97(\mathrm{Ar}-\mathrm{C}-\mathrm{OH}), 106.21(\mathrm{Ar}-\mathrm{C}-\mathrm{C}=\mathrm{O})$, 55.77,55.53 $\left(\mathrm{Ar}-\mathrm{OCH}_{3}\right), 7.26\left(\mathrm{Ar}-\mathrm{CH}_{3}\right), 161.25,160.45\left(\mathrm{Ar}^{1}-\right.$ C-F), 123.78,123.90 ( $\left.\mathrm{Ar}^{1}-\mathrm{C}\right), 131.18,131.09$ ( $\left.\mathrm{Ar}^{1}-\mathrm{C}\right), 130.79$, 130.72 ( $\left.\mathrm{Ar}^{1}-\mathrm{C}\right), 129.76,129.73\left(\mathrm{Ar}^{1}-\mathrm{C}\right), 116.33,116.11\left(\mathrm{Ar}^{1}{ }_{-}\right.$ C), $134.46(\mathrm{HC}=\mathrm{C}), 124.38(\mathrm{HC}=\mathrm{C})$.

Compound 3f. It is having the molecular formula $\mathrm{C}_{18} \mathrm{H}_{17} \mathrm{O}_{4} \mathrm{Br}$ which showed molecular ion peak in positive mode $(\mathrm{M}+\mathrm{H})^{+}$at 378 in LC-MS. So the molar mass of the compound $3 \mathrm{f}$ is $377 \mathrm{~g} / \mathrm{mol}$. Yield $-60 \%$, M.P- $175-180^{\circ} \mathrm{C}$.

${ }^{1} \mathrm{H} \mathrm{NMR}\left(\mathrm{CDCl}_{3}, 400 \mathrm{MHz}, \delta\right.$ in ppm): $1 \mathrm{H}, \mathrm{s} 13.99$ (Ar$\mathrm{OH}), 3 \mathrm{H}$, s $2.032\left(\mathrm{Ar}-\mathrm{CH}_{3}\right), 3 \mathrm{H}$, s $3.93\left(\mathrm{Ar}-\mathrm{OCH}_{3}\right), 3 \mathrm{H}, \mathrm{s}$ $3.89\left(\mathrm{Ar}^{-\mathrm{OCH}_{3}}\right), 1 \mathrm{H}, \mathrm{d}, 7.68(\mathrm{Ar}-\mathrm{H}), 2 \mathrm{H}, \mathrm{d}, 7.5\left(\mathrm{Ar}^{1}-\mathrm{H}\right), 2 \mathrm{H}$, $\mathrm{d}, 7.44\left(\mathrm{Ar}^{1}-\mathrm{H}\right), 1 \mathrm{H}, \mathrm{d}$, at $7.85(\mathrm{O}=\mathrm{C}-\mathrm{CH}=\mathrm{C}), 1 \mathrm{H}, \mathrm{d}, 5.98$ $(\mathrm{C}=\mathrm{C}-\mathrm{H})$.
TABLE 1: Media preparation for antituberculosis activity.

\begin{tabular}{lcc}
\hline Ml of stock $(\mathrm{mg} / \mathrm{L})$ & L J Fluid $(\mathrm{mL})$ & Final concentration $(\mathrm{mg} / \mathrm{L})$ \\
\hline $2 \mathrm{~mL}$ of 2000 & $20 \mathrm{~mL}$ & $200 \mathrm{mg} / \mathrm{L}$ \\
$1 \mathrm{~mL}$ of 2000 & $20 \mathrm{~mL}$ & $100 \mathrm{mg} / \mathrm{L}$ \\
$0.5 \mathrm{~mL}$ of 2000 & $20 \mathrm{~mL}$ & $50 \mathrm{mg} / \mathrm{L}$ \\
$0.25 \mathrm{~mL}$ of 2000 & $20 \mathrm{~mL}$ & $25 \mathrm{mg} / \mathrm{L}$ \\
$0.125 \mathrm{~mL}$ of 2000 & $20 \mathrm{~mL}$ & $12.5 \mathrm{mg} / \mathrm{L}$ \\
\hline
\end{tabular}

${ }^{13} \mathrm{C} \mathrm{NMR}\left(\mathrm{CDCl}_{3}, 100 \mathrm{MHz}, \delta\right.$ in $\left.\mathrm{ppm}\right): 192.70$ $(\mathrm{Ar}-\mathrm{C}=\mathrm{O}), \quad 7.23\left(\mathrm{Ar}-\mathrm{CH}_{3}\right), 106.26\left(\mathrm{Ar}-\mathrm{C}-\mathrm{CH}_{3}\right), 164.31$ $\left(\mathrm{Ar}-\mathrm{C}-\mathrm{OCH}_{3}\right), 86.42$ (Ar-C-H), 163.78 (Ar-C-OCH$)_{3}$, $106.33(\mathrm{Ar}-\mathrm{C}-\mathrm{C}=\mathrm{O}), 161.12(\mathrm{Ar}-\mathrm{C}-\mathrm{OH}), 55.84\left(\mathrm{Ar}-\mathrm{OCH}_{3}\right)$, $55.50\left(\mathrm{Ar}-\mathrm{O}-\mathrm{CH}_{3}\right), 134.68\left(\mathrm{Ar}^{1}-\mathrm{C}<\right), \delta$ at $129.60\left(\mathrm{Ar}^{1}-\mathrm{C}-\mathrm{H}\right)$, $128.65\left(\mathrm{Ar}^{1}-\mathrm{C}-\mathrm{H}\right), 132.08\left(\mathrm{Ar}^{1}-\mathrm{C}-\mathrm{Br}\right), 129.60\left(\mathrm{Ar}^{1}-\mathrm{C}-\mathrm{H}\right)$, 124.04 ( $\left.\mathrm{Ar}^{1}-\mathrm{C}-\mathrm{H}\right), 140.32(\mathrm{O}=\mathrm{C}-\mathrm{CH}=\mathrm{C}), 128.65(\mathrm{C}=\mathrm{C}-\mathrm{H})$.

Compound 3g. It is having the molecular formula $\mathrm{C}_{20} \mathrm{H}_{22} \mathrm{O}_{6}$ which showed molecular ion peak in negative mode $(\mathrm{M}-\mathrm{H})^{-}$at 357.2 so the molar mass of the compound $3 \mathrm{~g}$ is $358.2 \mathrm{~g} / \mathrm{mol}$. Yield $-75 \%$, M.P $-130-135^{\circ} \mathrm{C}$.

${ }^{1} \mathrm{H} \mathrm{NMR}\left(\mathrm{CDCl}_{3}, 400 \mathrm{MHz}, \delta\right.$ in ppm): $1 \mathrm{H}, \mathrm{s} 14.18$ (Ar$\mathrm{OH}), 3 \mathrm{H}, \mathrm{s} 2.03\left(\mathrm{Ar}-\mathrm{CH}_{3}\right), 6 \mathrm{H}$, s $3.9\left(\mathrm{Ar}-\mathrm{OCH}_{3}\right), 1 \mathrm{H}, \mathrm{s}, 5.98$ $(\mathrm{Ar}-\mathrm{H}), 1 \mathrm{H}, \mathrm{d}, 7.21(\mathrm{O}=\mathrm{C}-\mathrm{CH}=\mathrm{C}), 1 \mathrm{H}, \mathrm{d}, 6.89(\mathrm{C}=\mathrm{C}-\mathrm{H}), 2 \mathrm{H}$, $\mathrm{m}, 7.7\left(\mathrm{Ar}^{1}-\mathrm{H}\right), 1 \mathrm{H}, \mathrm{s} 7.11\left(\mathrm{Ar}^{1}-\mathrm{H}\right), 3 \mathrm{H}, \mathrm{s} 3.92\left(\mathrm{Ar}^{1}-\mathrm{OCH}_{3}\right)$, $3 \mathrm{H}, \mathrm{s}, 3.89\left(\mathrm{Ar}^{1}-\mathrm{OCH}_{3}\right)$.

${ }^{13} \mathrm{CNMR}\left(100 \mathrm{MHz}, \delta\right.$ in ppm, $\left.\mathrm{CDCl}_{3}\right): 192.85$ (Ar-C=O), $106.39\left(\mathrm{Ar}-\mathrm{C}-\mathrm{CH}_{3}\right), 164.27\left(\mathrm{Ar}-\mathrm{C}-\mathrm{OCH}_{3}\right), 163.43$ (Ar-C$\left.\mathrm{OCH}_{3}\right), 160.99(\mathrm{Ar}-\mathrm{C}-\mathrm{OH}), 86.46(\mathrm{Ar}-\mathrm{C}-\mathrm{H}), 106.20(\mathrm{Ar}-$ $\mathrm{C}-\mathrm{C}=\mathrm{O}), 55.98\left(\mathrm{Ar}-\mathrm{OCH}_{3}\right) 55.89\left(\mathrm{Ar}-\mathrm{O}-\mathrm{CH}_{3}\right), 125.92\left(\mathrm{Ar}^{1}-\right.$ $\mathrm{C}<), 110.65$ ( $\left.\mathrm{Ar}^{1}-\mathrm{C}-\mathrm{H}\right), 111.31\left(\mathrm{Ar}^{1}-\mathrm{C}-\mathrm{H}\right), 122.52\left(\mathrm{Ar}^{1}-\mathrm{C}-\right.$ $\mathrm{H}), 151.07\left(\mathrm{Ar}^{1}-\mathrm{C}-\mathrm{OCH}_{3}\right), 149.22\left(\mathrm{Ar}^{1}-\mathrm{C}-\mathrm{OCH}_{3}\right), 55.79$ $\left(\mathrm{Ar}^{1}-\mathrm{OCH}_{3}\right), 55.47\left(\mathrm{Ar}^{1}-\mathrm{OCH}_{3}\right), 142.18(\mathrm{CH}=\mathrm{C}), 128.52$ $(\mathrm{C}=\mathrm{C}-\mathrm{H}) .7 .25\left(\mathrm{Ar}-\mathrm{CH}_{3}\right)$.

Compound $3 \mathbf{h}$. It is having the molecular formula $\mathrm{C}_{18} \mathrm{H}_{16} \mathrm{O}_{4} \mathrm{~F}_{2}$ which showed molecular ion peak in negative mode $(\mathrm{M}-\mathrm{H})^{-}$at 333.2 in LC-MS. So the molar mass of the compound $3 \mathrm{~h}$ is $334.2 \mathrm{~g} / \mathrm{mol}$. Yield $-60 \%$, M.P- $135-140^{\circ} \mathrm{C}$.

${ }^{1} \mathrm{H}$ NMR $\left(\mathrm{CDCl}_{3}, 400 \mathrm{MHz}, \delta\right.$ in ppm): $3 \mathrm{H}, \mathrm{s}, 2.03$ (Ar$\left.\mathrm{CH}_{3}\right), 3 \mathrm{H}, \mathrm{s} 3.95\left(\mathrm{Ar}-\mathrm{OCH}_{3}\right), 3 \mathrm{H}$, s $3.90(\mathrm{Ar}-\mathrm{H}), 1 \mathrm{H}, \mathrm{s}, 7.78$ $\left(\mathrm{Ar}-\mathrm{OCH}_{3}\right), 1 \mathrm{H}, \mathrm{s} 13.94(\mathrm{Ar}-\mathrm{OH}), 1 \mathrm{H}, \mathrm{d}, 6.89(\mathrm{O}=\mathrm{C}-\mathrm{CH}=\mathrm{C})$, 1H, d, $7.21(\mathrm{C}=\mathrm{C}-\mathrm{H}), 1 \mathrm{H}, \mathrm{m}, 7.7\left(\mathrm{Ar}^{1}-\mathrm{H}\right), 1 \mathrm{H}, \mathrm{m} 7.3\left(\mathrm{Ar}^{1}-\mathrm{H}\right)$, $1 \mathrm{H}, \mathrm{m}, 7.1\left(\mathrm{Ar}^{1}-\mathrm{H}\right)$.

${ }^{13} \mathrm{C} \mathrm{NMR}\left(\mathrm{CDCl}_{3}, 100 \mathrm{MHz}, \delta\right.$ in $\left.\mathrm{ppm}\right): 7.78$ (Ar$\left.\mathrm{CH}_{3}\right), 106.30\left(\mathrm{Ar}-\mathrm{C}-\mathrm{CH}_{3}\right), 163.86\left(\mathrm{Ar}-\mathrm{C}-\mathrm{OCH}{ }_{3}\right), 86.42(\mathrm{Ar}-$ $\mathrm{C}-\mathrm{H}), 164.31$ (Ar-C-OCH$\left.{ }_{3}\right), 106.27$ (Ar-C-C=O), 161.12 $(\mathrm{Ar}-\mathrm{C}-\mathrm{OH}), 55.86\left(\mathrm{Ar}-\mathrm{OCH}_{3}\right), 55.47\left(\mathrm{Ar}-\mathrm{O}-\mathrm{CH}_{3}\right), 192.69$ $(\mathrm{Ar}-\mathrm{C}=\mathrm{O}), 129.0\left(\mathrm{Ar}^{1}-\mathrm{C}<\right), 124.99\left(\mathrm{Ar}^{1}-\mathrm{C}-\mathrm{H}\right), 125.06\left(\mathrm{Ar}^{1}-\right.$ C-H), 133.06 (Ar $\left.{ }^{1}-\mathrm{C}-\mathrm{F}\right), 132.98$ (Ar $\left.{ }^{1}-\mathrm{C}-\mathrm{F}\right), 117.8$ (Ar $\left.{ }^{1}-\mathrm{C}-\mathrm{H}\right)$, 139.26 $(\mathrm{CH}=\mathrm{C}), 126.81(\mathrm{C}=\mathrm{C}-\mathrm{H})$.

3.1.7. General Method for Synthesis of Flavanones (Compound $\mathbf{4 a - h})$. To the previously obtained chalcones (3a-h) (0.2 g) was refluxed in alcoholic sulphuric acid $(10 \%, 10 \mathrm{~mL})$ for $24 \mathrm{~h}$. The alcohol was distilled off in vacuo and the residue is diluted with water. The separated product was dissolved 
in ethyl acetate and dried over sodium sulphate. The ethyl acetate layer was concentrated the residue was washed with ether to remove any adherent chalcone to obtain pure flavanones $(\mathbf{4 a}-\mathbf{h})$.

Compound 4a. It is having the molecular formula $\mathrm{C}_{19} \mathrm{H}_{20} \mathrm{O}_{5}$ which showed molecular ion peak in positive mode $(\mathrm{M}+\mathrm{H})^{+}$at 329 in LC-MS. So the molar mass of the compound $4 \mathrm{a}$ is $328 \mathrm{~g} / \mathrm{mol}$. Yield $-70 \%$, M.P $-172-175^{\circ} \mathrm{C}$.

${ }^{1} \mathrm{H}$ NMR: $\left(\mathrm{CDCl}_{3}, 400 \mathrm{MHz}, \delta\right.$ in ppm): $1 \mathrm{H}, \mathrm{s}, 6.27(\mathrm{Ar}-$ $\mathrm{H}), 1 \mathrm{H}, \mathrm{m}, 7.19\left(\mathrm{Ar}^{1}-\mathrm{H}\right), 2 \mathrm{H}, \mathrm{m}, 6.7\left(\mathrm{Ar}^{1}-\mathrm{H}\right), 1 \mathrm{H}, \mathrm{d}, 6.8\left(\mathrm{Ar}^{1}-\right.$ $\mathrm{H}), 1 \mathrm{H}, \mathrm{d}, 4.82(\mathrm{O}-\mathrm{C}-\mathrm{H}), 6 \mathrm{H}, \mathrm{s}, 3.86\left(\mathrm{Ar}-\mathrm{O}-\mathrm{CH}_{3}\right), 3.71(3 \mathrm{H}, \mathrm{s}$, $\left.\mathrm{Ar}^{1}-\mathrm{O}-\mathrm{CH}_{3}\right), 3 \mathrm{H}, \mathrm{s}, 2.16\left(\mathrm{Ar}-\mathrm{CH}_{3}\right), 3.06$ and $2.88(1 \mathrm{H}, \mathrm{d}$ and $1 \mathrm{H}, \mathrm{d} \mathrm{O}=\mathrm{C}-\mathrm{CH}_{2}$ ).

${ }^{13} \mathrm{C}$ NMR: $\left(\mathrm{CDCl}_{3}, 100 \mathrm{MHz}, \delta\right.$ in ppm): $7.81\left(\mathrm{Ar}-\mathrm{CH}_{3}\right)$, $106.36\left(\mathrm{Ar}-\mathrm{C}-\mathrm{CH}_{3}\right), 163.56\left(\mathrm{Ar}-\mathrm{C}-\mathrm{OCH}_{3}\right), 88.64(\mathrm{Ar}-\mathrm{C}-\mathrm{H})$, $160.12\left(\mathrm{Ar}-\mathrm{C}-\mathrm{OCH}_{3}\right), 105.98(\mathrm{Ar}-\mathrm{C}-\mathrm{C}=\mathrm{O}), 160.52(\mathrm{Ar}-\mathrm{C}-$ $\mathrm{O}), 55.92\left(\mathrm{Ar}-\mathrm{OCH}_{3}\right), 55.08\left(\mathrm{Ar}-\mathrm{O}-\mathrm{CH}_{3}\right), 189.83(\mathrm{Ar}-\mathrm{C}=\mathrm{O})$, $129.73\left(\mathrm{Ar}^{1}-\mathrm{C}<\right), 118.01\left(\mathrm{Ar}^{1}-\mathrm{C}-\mathrm{H}\right), 159.90\left(\mathrm{Ar}^{1}-\mathrm{C}-\mathrm{OCH}_{3}\right)$, $111.58\left(\mathrm{Ar}^{1}-\mathrm{C}-\mathrm{H}\right), 140.99\left(\mathrm{Ar}^{1}-\mathrm{C}-\mathrm{H}\right), 113.63\left(\mathrm{Ar}^{1}-\mathrm{C}-\mathrm{H}\right)$, $55.62\left(\mathrm{Ar}^{1}-\mathrm{OCH}_{3}\right), 45.75\left(\mathrm{O}=\mathrm{C}-\mathrm{CH}_{2}\right), 78.28(>\mathrm{C}-\mathrm{H})$.

Compound $\mathbf{4 b}$. It is having the molecular formula $\mathrm{C}_{19} \mathrm{H}_{20} \mathrm{O}_{5}$ which showed molecular ion peak in positive mode $(\mathrm{M}+\mathrm{H})^{+}$at 329 in LC-MS. So the molar mass of the compound $4 \mathrm{~b}$ is $328 \mathrm{~g} / \mathrm{mol}$. Yield $-60 \%$, M.P $-166-170^{\circ} \mathrm{C}$.

${ }^{1} \mathrm{H}$ NMR: ( $\mathrm{CDCl}_{3}, 400 \mathrm{MHz}, \delta$ in ppm): $1 \mathrm{H}, \mathrm{s}, 6.125$ (Ar$\mathrm{H}), 2 \mathrm{H}, \mathrm{s}, 7.04\left(\mathrm{Ar}^{1}-\mathrm{H}\right), 1 \mathrm{H}, \mathrm{dd}, 6.8\left(\mathrm{Ar}^{1}-\mathrm{H}\right), 1 \mathrm{H}, \mathrm{dd}, 6.98$ (Ar-H), 1H, dd, $5.3(\mathrm{O}-\mathrm{C}-\mathrm{H}), 3 \mathrm{H}, \mathrm{s}, 3.93\left(\mathrm{Ar}-\mathrm{O}-\mathrm{CH}_{3}\right), 3 \mathrm{H}, \mathrm{s}$, $3.93\left(\mathrm{Ar}-\mathrm{O}-\mathrm{CH}_{3}\right), 3 \mathrm{H}, \mathrm{s}, 3.83\left(\mathrm{Ar}^{1}-\mathrm{O}-\mathrm{CH}_{3}\right), 3 \mathrm{H}, \mathrm{s}, 2.06(\mathrm{Ar}-$ $\left.\mathrm{CH}_{3}\right), 2.9$, and $2.8\left(1 \mathrm{H}\right.$, dd and $1 \mathrm{H}$, dd, $\left.\mathrm{O}=\mathrm{C}-\mathrm{CH}_{2}\right)$.

${ }^{13} \mathrm{C} \mathrm{NMR:}\left(\mathrm{CDCl}_{3}, 100 \mathrm{MHz}, \delta\right.$ in ppm): $7.81\left(\mathrm{Ar}-\mathrm{CH}_{3}\right)$, $106.3\left(\mathrm{Ar}-\mathrm{C}-\mathrm{CH}_{3}\right), 161.12\left(\mathrm{Ar}-\mathrm{C}-\mathrm{OCH}_{3}\right), 88.64(\mathrm{Ar}-\mathrm{C}-\mathrm{H})$, $160.52\left(\mathrm{Ar}-\mathrm{C}-\mathrm{OCH}_{3}\right), 105.98(\mathrm{Ar}-\mathrm{C}-\mathrm{C}=\mathrm{O}), 163.56(\mathrm{Ar}-\mathrm{C}-$ $\mathrm{O}), 55.62\left(\mathrm{Ar}-\mathrm{OCH}_{3}\right), 56.06\left(\mathrm{Ar}-\mathrm{O}-\mathrm{CH}_{3}\right), 189.83(\mathrm{Ar}-\mathrm{C}=\mathrm{O})$, $140.99\left(\mathrm{Ar}^{1}-\mathrm{C}<\right), 129.97\left(\mathrm{Ar}^{1}-\mathrm{C}-\mathrm{H}\right), 159.90\left(\mathrm{Ar}^{1}-\mathrm{C}-\mathrm{OCH}_{3}\right)$, $118.01\left(\mathrm{Ar}^{1}-\mathrm{C}-\mathrm{H}\right), 111.58\left(\mathrm{Ar}^{1}-\mathrm{C}-\mathrm{H}\right), 113.6\left(\mathrm{Ar}^{1}-\mathrm{C}-\mathrm{H}\right), 55.27$ $\left(\mathrm{Ar}^{1}-\mathrm{OCH}_{3}\right), 45.75\left(\mathrm{O}=\mathrm{C}-\mathrm{CH}_{2}\right), 78.28(>\mathrm{C}-\mathrm{H})$.

Compound 4c. It is having the molecular formula $\mathrm{C}_{21} \mathrm{H}_{24} \mathrm{O}_{7}$ which showed molecular ion peak in positive mode $(\mathrm{M}+\mathrm{Na})^{+}$at 411.2 in LC-MS. So the molar mass of the compound $4 \mathrm{c}$ is $388.2 \mathrm{~g} / \mathrm{mol}$. Yield $-65 \%$, M.P- $164-170^{\circ} \mathrm{C}$.

${ }^{1} \mathrm{H}$ NMR: $\left(\mathrm{CDCl}_{3}, 400 \mathrm{MHz}, \delta\right.$ in ppm): $1 \mathrm{H}, \mathrm{s}, 6.13(\mathrm{Ar}-$ $\mathrm{C}-\mathrm{H}), 3 \mathrm{H}, \mathrm{s}, 2.06\left(\mathrm{Ar}^{-\mathrm{CH}_{3}}\right), 2 \mathrm{H}, \mathrm{s}, 6.69\left(\mathrm{Ar}^{1}-\mathrm{C}-\mathrm{H}\right), 1 \mathrm{H}, \mathrm{dd}$, $5.3\left(\mathrm{Ar}^{1}-\mathrm{O}-\mathrm{C}-\mathrm{H}\right), 3 \mathrm{H}, \mathrm{s}, 3.94\left(\mathrm{Ar}^{-} \mathrm{OCH}_{3}\right), 3 \mathrm{H}, \mathrm{s}, 3.91(\mathrm{Ar}-$ $\left.\mathrm{OCH}_{3}\right), 3 \mathrm{H}, \mathrm{s}, 3.8\left(\mathrm{Ar}^{1}-\mathrm{OCH}_{3}\right), 6 \mathrm{H}, \mathrm{s}, 3.88\left(\mathrm{Ar}^{1}-\mathrm{OCH}_{3}\right), 1 \mathrm{H}$, dd at $\delta 2.97$ and $1 \mathrm{H}$, dd at $\delta 2.86(-\mathrm{O}=\mathrm{C}-\mathrm{CH} 2-)$.

${ }^{13} \mathrm{C} \mathrm{NMR:}\left(\mathrm{CDCl}_{3}, 100 \mathrm{MHz}, \delta\right.$ in ppm): $7.83\left(\mathrm{Ar}-\mathrm{CH}_{3}\right)$, $105.91\left(\mathrm{Ar}-\mathrm{C}-\mathrm{CH}_{3}\right), 161.04\left(\mathrm{Ar}-\mathrm{C}-\mathrm{OCH}_{3}\right), 88.62(\mathrm{Ar}-\mathrm{C}-\mathrm{H})$, $160.54\left(\mathrm{Ar}-\mathrm{C}-\mathrm{OCH}_{3}\right), 103.03(\mathrm{Ar}-\mathrm{C}-\mathrm{C}=\mathrm{O}), 163.61(\mathrm{Ar}-\mathrm{C}-$ $\mathrm{O}), 56.20\left(\mathrm{Ar}-\mathrm{OCH}_{3}\right), 56.06\left(\mathrm{Ar}-\mathrm{O}-\mathrm{CH}_{3}\right), 189.85(\mathrm{Ar}-\mathrm{C}=\mathrm{O})$, $138.01\left(\mathrm{Ar}^{1}-\mathrm{C}<\right), 103.03\left(\mathrm{Ar}^{1}-\mathrm{C}-\mathrm{H}\right), 153.47\left(\mathrm{Ar}^{1}-\mathrm{C}-\mathrm{OCH}_{3}\right)$, $153.47\left(\mathrm{Ar}^{1}-\mathrm{C}-\mathrm{OCH}_{3}\right), 134.96\left(\mathrm{Ar}^{1}-\mathrm{C}-\mathrm{OCH}_{3}\right), 103.03\left(\mathrm{Ar}^{1}-\right.$ $\mathrm{C}-\mathrm{H}), 55.64\left(\mathrm{Ar}^{1}-\mathrm{OCH}_{3}\right), 55.64\left(\mathrm{Ar}^{1}-\mathrm{OCH}_{3}\right), 60.83\left(\mathrm{Ar}^{1}{ }^{-}\right.$ $\left.\mathrm{OCH}_{3}\right), 45.78\left(\mathrm{O}=\mathrm{C}-\mathrm{CH}_{2}\right), 78.42(>\mathrm{C}-\mathrm{H})$.

Compound 4d. It is having the molecular formula $\mathrm{C}_{18} \mathrm{H}_{17} \mathrm{O}_{4} \mathrm{Cl}$ which showed molecular ion peak in positive mode $(\mathrm{M}+\mathrm{H})^{+}$at 333.2 in LC-MS. So the molar mass of the compound $4 \mathrm{~d}$ is $332 \mathrm{~g} / \mathrm{mol}$. Yield-60\%, M.P- $177-180^{\circ} \mathrm{C}$.

${ }^{1} \mathrm{HNMR}\left(400 \mathrm{MHz}, \delta\right.$ in ppm, $\left.\mathrm{CDCl}_{3}\right): 3 \mathrm{H}, \mathrm{s}, 2.04(\mathrm{Ar}-$ $\left.\mathrm{CH}_{3}\right), 3 \mathrm{H}, \mathrm{s}, 3.93\left(\mathrm{Ar}-\mathrm{OCH}_{3}\right), 3 \mathrm{H}, \mathrm{s}, 3.90\left(\mathrm{Ar}-\mathrm{OCH}_{3}\right), 1 \mathrm{H}, \mathrm{s}$, $6.128(\mathrm{Ar}-\mathrm{H}), 2 \mathrm{H}, \mathrm{m}, 2.9\left(\mathrm{O}=\mathrm{C}-\mathrm{CH}_{2}\right), 1 \mathrm{H}, \mathrm{dd}, 5.38(>\mathrm{C}<\mathrm{H})$, $4 \mathrm{H}, \mathrm{m}, 7.4\left(\mathrm{Ar}^{1}-\mathrm{H}\right)$.

${ }^{13} \mathrm{C}$ NMR: $\left(\mathrm{CDCl}_{3}, 100 \mathrm{MHz}, \delta\right.$ in ppm): $7.79\left(\mathrm{Ar}^{-\mathrm{CH}_{3}}\right)$, $106.33\left(\mathrm{Ar}-\mathrm{C}-\mathrm{CH}_{3}\right), 160.87\left(\mathrm{Ar}-\mathrm{C}-\mathrm{OCH}_{3}\right), 88.69(\mathrm{Ar}-\mathrm{C}-\mathrm{H})$, $160.58\left(\mathrm{Ar}-\mathrm{C}-\mathrm{OCH}_{3}\right), 105.33(\mathrm{Ar}-\mathrm{C}-\mathrm{C}=\mathrm{O}), 163.67(\mathrm{Ar}-\mathrm{C}-$ $\mathrm{O}), 55.63\left(\mathrm{Ar}-\mathrm{OCH}_{3}\right), 56.06\left(\mathrm{Ar}-\mathrm{O}-\mathrm{CH}_{3}\right), 189.47(\mathrm{Ar}-\mathrm{C}=\mathrm{O})$, 134.16 ( $\left.\mathrm{Ar}^{1}-\mathrm{C}<\right), 127.21\left(\mathrm{Ar}^{1}-\mathrm{C}-\mathrm{H}\right), 128.8\left(\mathrm{Ar}^{1}-\mathrm{C}-\mathrm{H}\right), 137.87$ $\left(\mathrm{Ar}^{1}-\mathrm{C}-\mathrm{Cl}\right), 128.88\left(\mathrm{Ar}^{1}-\mathrm{C}-\mathrm{H}\right), 127.21\left(\mathrm{Ar}^{1}-\mathrm{C}-\mathrm{H}\right), 45.56$ $\left(\mathrm{O}=\mathrm{C}-\mathrm{CH}_{2}\right), 77.74(>\mathrm{C}-\mathrm{H})$.

Compound 4e. It is having the molecular formula $\mathrm{C}_{18} \mathrm{H}_{17} \mathrm{O}_{4} \mathrm{~F}$ which showed molecular ion peak in positive mode $(\mathrm{M}+\mathrm{H})^{+}$at 317.3 in LC-MS. So the molar mass of the compound $4 \mathrm{e}$ is $316.3 \mathrm{~g} / \mathrm{mol}$. Yield $-65 \%$, M.P- $169-175^{\circ} \mathrm{C}$.

${ }^{1} \mathrm{H}$ NMR: $\left(\mathrm{CDCl}_{3}, 400 \mathrm{MHz}, \delta\right.$ in ppm): $3 \mathrm{H}, \mathrm{s}, 2.04$ (Ar$\left.\mathrm{CH}_{3}\right), 3 \mathrm{H}, \mathrm{s}, 3.94\left(\mathrm{Ar}-\mathrm{OCH}_{3}\right), 3 \mathrm{H}, \mathrm{s}, 3.90\left(\mathrm{Ar}-\mathrm{OCH}_{3}\right), 1 \mathrm{H}, \mathrm{s}$, $6.14(\mathrm{Ar}-\mathrm{H}), 2 \mathrm{H}, \mathrm{m}, 2.9\left(\mathrm{O}=\mathrm{C}-\mathrm{CH}_{2}\right), 1 \mathrm{H}, \mathrm{dd}, 5.69(>\mathrm{C}<\mathrm{H})$, $1 \mathrm{H}, \mathrm{m}, 7.6\left(\mathrm{Ar}^{1}-\mathrm{H}\right), 1 \mathrm{H}, \mathrm{m}, 7.3\left(\mathrm{Ar}^{1}-\mathrm{H}\right), 1 \mathrm{H}, \mathrm{m}, 7.2\left(\mathrm{Ar}^{1}-\mathrm{H}\right)$, $1 \mathrm{H}, \mathrm{m}, 7.1\left(\mathrm{Ar}^{1}-\mathrm{H}\right)$.

${ }^{13} \mathrm{C}$ NMR: $\left(\mathrm{CDCl}_{3}, 100 \mathrm{MHz}, \delta\right.$ in ppm) $7.74\left(\mathrm{Ar}-\mathrm{CH}_{3}\right)$, $106.41\left(\mathrm{Ar}-\mathrm{C}-\mathrm{CH}_{3}\right), 161.13\left(\mathrm{Ar}-\mathrm{C}-\mathrm{OCH}_{3}\right), 88.80(\mathrm{Ar}-\mathrm{C}-\mathrm{H})$, $160.62\left(\mathrm{Ar}-\mathrm{C}-\mathrm{OCH}_{3}\right), 105.97(\mathrm{Ar}-\mathrm{C}-\mathrm{C}=\mathrm{O}), 163.56(\mathrm{Ar}-\mathrm{C}-$ $\mathrm{O}), 56.10\left(\mathrm{Ar}-\mathrm{OCH}_{3}\right), 55.64\left(\mathrm{Ar}-\mathrm{O}-\mathrm{CH}_{3}\right), 189.50(\mathrm{Ar}-\mathrm{C}=\mathrm{O})$, $115.5\left(\mathrm{Ar}^{1}-\mathrm{C}<\right), 126.8\left(\mathrm{Ar}^{1}-\mathrm{C}-\mathrm{H}\right), 129.8\left(\mathrm{Ar}^{1}-\mathrm{C}-\mathrm{H}\right), 160.96$, $\left(\mathrm{Ar}^{1}-\mathrm{C}-\mathrm{F}\right), 127.15$ ( $\left.\mathrm{Ar}^{1}-\mathrm{C}-\mathrm{H}\right), 124.4 \quad\left(\mathrm{Ar}^{1}-\mathrm{C}-\mathrm{H}\right), 44.73$ $\left(\mathrm{O}=\mathrm{C}-\mathrm{CH}_{2}\right), 73.14(>\mathrm{C}-\mathrm{H})$.

Compound $\mathbf{4 f}$. It is having the molecular formula $\mathrm{C}_{18} \mathrm{H}_{17} \mathrm{O}_{4} \mathrm{Br}$ which showed molecular ion peak in positive mode $(\mathrm{M}+\mathrm{H})^{+}$at 378.1 in LC-MS. So the molar mass of the compound $4 \mathrm{f}$ is $377 \mathrm{~g} / \mathrm{mol}$. Yield $-60 \%$, M.P- $166-170^{\circ} \mathrm{C}$.

${ }^{1} \mathrm{H}$ NMR: $\left(\mathrm{CDCl}_{3}, 400 \mathrm{MHz}, \delta\right.$ in ppm): $3 \mathrm{H}, \mathrm{s}, 2.04$ (Ar$\left.\mathrm{CH}_{3}\right), 3 \mathrm{H}, \mathrm{s}, 3.93\left(\mathrm{Ar}-\mathrm{OCH}_{3}\right), 3 \mathrm{H}, \mathrm{s} 3.90\left(\mathrm{Ar}-\mathrm{OCH}_{3}\right), 1 \mathrm{H}, \mathrm{s}$, $6.12(\mathrm{Ar}-\mathrm{H}), 2 \mathrm{H}, \mathrm{m}, 2.8\left(\mathrm{O}=\mathrm{C}-\mathrm{CH}_{2}\right), 1 \mathrm{H}, \mathrm{dd}, 5.3\left(>\mathrm{C}<_{\mathrm{H}}\right), 2 \mathrm{H}$, d, $7.5\left(\mathrm{Ar}^{1}-\mathrm{H}\right), 2 \mathrm{H}, \mathrm{d}, 7.3\left(\mathrm{Ar}^{1}-\mathrm{H}\right)$.

${ }^{13} \mathrm{C}$ NMR: $\left(\mathrm{CDCl}_{3}, 100 \mathrm{MHz}, \delta\right.$ in ppm): $7.78(\mathrm{Ar}-$ $\left.\mathrm{CH}_{3}\right), 106.31\left(\mathrm{Ar}-\mathrm{C}-\mathrm{CH}_{3}\right), 160.84\left(\mathrm{Ar}-\mathrm{C}-\mathrm{OCH}_{3}\right), 88.73(\mathrm{Ar}-$ $\mathrm{C}-\mathrm{H}), 160.56\left(\mathrm{Ar}-\mathrm{C}-\mathrm{OCH}_{3}\right), 105.89(\mathrm{Ar}-\mathrm{C}-\mathrm{C}=\mathrm{O}), 163.64$ (Ar-C-O), $56.05\left(\mathrm{Ar}-\mathrm{OCH}_{3}\right), 55.63\left(\mathrm{Ar}-\mathrm{O}-\mathrm{CH}_{3}\right), 189.33$ $(\mathrm{Ar}-\mathrm{C}=\mathrm{O}), 131.84\left(\mathrm{Ar}^{1}-\mathrm{C}<\right), 122.24\left(\mathrm{Ar}^{1}-\mathrm{C}-\mathrm{H}\right), 127.51\left(\mathrm{Ar}^{1}-\right.$ $\mathrm{C}-\mathrm{H}), 138.41\left(\mathrm{Ar}^{1}-\mathrm{C}-\mathrm{Br}\right), 127.51\left(\mathrm{Ar}^{1}-\mathrm{C}-\mathrm{H}\right), 122.24\left(\mathrm{Ar}^{1}-\mathrm{C}-\right.$ $\mathrm{H}), 45.50\left(\mathrm{O}=\mathrm{C}-\mathrm{CH}_{2}\right), 77.77(>\mathrm{C}-\mathrm{H})$.

Compound 4g. It is having the molecular formula $\mathrm{C}_{20} \mathrm{H}_{22} \mathrm{O}_{6}$ which showed molecular ion peak in positive mode $(\mathrm{M}+\mathrm{Na})^{+}$at 381.3 in LC-MS. So the molar mass of the compound $4 \mathrm{~g}$ is $359.3 \mathrm{~g} / \mathrm{mol}$. Yield $-75 \%$, M.P $-179-182^{\circ} \mathrm{C}$.

${ }^{1} \mathrm{HNMR}\left(400 \mathrm{MHz}, \delta\right.$ in ppm, $\left.\mathrm{CDCl}_{3}\right): 1 \mathrm{H}, \mathrm{s}, 6.13(\mathrm{Ar}-$ $\mathrm{C}-\mathrm{H}), 3 \mathrm{H}, \mathrm{s}, 2.06\left(\mathrm{Ar}^{-\mathrm{CH}_{3}}\right), 2 \mathrm{H}, \mathrm{s}, 7.0\left(\mathrm{Ar}^{1}-\mathrm{C}-\mathrm{H}\right), 1 \mathrm{H}, \mathrm{d}, 6.98$ $\left(\mathrm{Ar}^{1}-\mathrm{C}-\mathrm{H}\right), 1 \mathrm{H}, \mathrm{dd}, 5.3\left(\mathrm{Ar}^{1}-\mathrm{O}-\mathrm{C}-\mathrm{H}\right), 3 \mathrm{H}, \mathrm{s}, 3.93\left(\mathrm{Ar}-\mathrm{OCH}_{3}\right)$, $9 \mathrm{H}, \mathrm{s}, 3.90\left(\mathrm{Ar}^{-} \mathrm{OCH}_{3}\right), 2\left(\mathrm{Ar}^{1}-\mathrm{OCH} 3\right), 1 \mathrm{H}$, dd and $1 \mathrm{H}$, dd, 2.97 and $2.86\left(-\mathrm{O}=\mathrm{C}-\mathrm{CH}_{2}-\right)$.

${ }^{13} \mathrm{C}$ NMR: $\left(\mathrm{CDCl}_{3}, 100 \mathrm{MHz}, \delta\right.$ in ppm) $7.83(\mathrm{Ar}-$ $\left.\mathrm{CH}_{3}\right), 106.29\left(\mathrm{Ar}-\mathrm{C}-\mathrm{CH}_{3}\right), 161.17\left(\mathrm{Ar}-\mathrm{C}-\mathrm{OCH}_{3}\right), 88.59(\mathrm{Ar}-$ $\mathrm{C}-\mathrm{H}), 160.52\left(\mathrm{Ar}-\mathrm{C}-\mathrm{OCH}_{3}\right), 106.29(\mathrm{Ar}-\mathrm{C}-\mathrm{C}=\mathrm{O}), 160.52$ 
TABLE 2: Antioxidant and cytotoxic activity of chalcones (Compounds 3a-h).

\begin{tabular}{|c|c|c|c|c|c|c|c|}
\hline \multirow{2}{*}{ Test name } & \multicolumn{3}{|c|}{ Superoxide inhibitory activity } & \multicolumn{3}{|c|}{ DPPH inhibitory activity } & \multirow{2}{*}{$\begin{array}{l}\text { Brine shrimp Lethality test } \\
\qquad \mathrm{ED}_{50}\end{array}$} \\
\hline & Dose at $(\mu \mathrm{g} / \mathrm{mL})$ & $\%$ of inhibition & $\mathrm{IC}_{50}$ & Dose at $(\mu \mathrm{g} / \mathrm{mL})$ & $\%$ of inhibition & $\mathrm{IC}_{50}$ & \\
\hline \multirow{3}{*}{ Compound-3a } & 50 & 4.58 & \multirow{3}{*}{93.89} & 50 & 8.36 & \multirow{3}{*}{85.74} & \multirow{3}{*}{46.56} \\
\hline & 75 & 28.92 & & 75 & 32.12 & & \\
\hline & 100 & 57.03 & & 100 & 69.94 & & \\
\hline \multirow{3}{*}{ Compound-3b } & 50 & 7.35 & \multirow{3}{*}{90.95} & 50 & 20.73 & \multirow{3}{*}{78.49} & \multirow{3}{*}{56.45} \\
\hline & 75 & 25.33 & & 75 & 47.27 & & \\
\hline & 100 & 63.56 & & 100 & 71.52 & & \\
\hline \multirow{3}{*}{ Compound-3c } & 50 & 18.63 & \multirow{3}{*}{86.58} & 50 & 23.15 & \multirow{3}{*}{75.42} & \multirow{3}{*}{63.29} \\
\hline & 75 & 36.76 & & 75 & 50.06 & & \\
\hline & 100 & 69.44 & & 100 & 75.52 & & \\
\hline \multirow{3}{*}{ Compound-3d } & 50 & 25.82 & \multirow{3}{*}{85.52} & 50 & 20.61 & \multirow{3}{*}{77.29} & \multirow{3}{*}{32.45} \\
\hline & 100 & 45.42 & & 75100 & & & \\
\hline & 150 & 79.25 & & 75100 & $4 / .64 / 4.42$ & & \\
\hline \multirow{3}{*}{ Compound-3e } & 25 & 30.88 & \multirow{3}{*}{83.27} & 50 & 28.97 & \multirow{3}{*}{74.90} & \multirow{3}{*}{28.34} \\
\hline & 50 & 47.06 & & 75 & 49.94 & & \\
\hline & 75 & 78.43 & & 100 & 71.52 & & \\
\hline \multirow{3}{*}{ Compound-3f } & 50 & 31.05 & \multirow{3}{*}{80.15} & 50 & 20.48 & \multirow{3}{*}{91.14} & \multirow{3}{*}{35.26} \\
\hline & 75 & 61.76 & & 75 & 36.97 & & \\
\hline & 100 & 76.63 & & 100 & 57.09 & & \\
\hline \multirow{3}{*}{ Compound-3g } & 50 & 12.75 & \multirow{3}{*}{86.58} & 50 & 23.15 & \multirow{3}{*}{75.42} & \\
\hline & 75 & 33.01 & & 75 & 50.06 & & 38.56 \\
\hline & 100 & 66.83 & & 100 & 75.52 & & \\
\hline & 25 & 6.05 & & 50 & 20.61 & & \\
\hline Compound-3h & 50 & 37.09 & 85.52 & 75 & 47.64 & 77.29 & 25.36 \\
\hline & 75 & 56.37 & & 100 & 74.42 & & \\
\hline & 2.5 & 34.49 & & 2.5 & 34.49 & & \\
\hline Vit-C & 5 & 62.71 & 3.98 & 5 & 62.71 & 3.98 & Phodophyllotoxin 3.69 \\
\hline & 10 & 102.27 & & 10 & 102.27 & & \\
\hline
\end{tabular}

(Ar-C-O), $56.07\left(\mathrm{Ar}-\mathrm{OCH}_{3}\right), 55.99\left(\mathrm{Ar}-\mathrm{O}-\mathrm{CH}_{3}\right), 190.03$ $(\mathrm{Ar}-\mathrm{C}=\mathrm{O}), 131.93\left(\mathrm{Ar}^{1}-\mathrm{C}<\right), 109.40\left(\mathrm{Ar}^{1}-\mathrm{C}-\mathrm{H}\right), 118.38\left(\mathrm{Ar}^{1}-\right.$ $\mathrm{C}-\mathrm{H}), 149.15\left(\mathrm{Ar}^{1}-\mathrm{C}-\mathrm{OCH}_{3}\right), 149.23\left(\mathrm{Ar}^{1}-\mathrm{C}-\mathrm{OCH}_{3}\right), 111.27$ $\left(\mathrm{Ar}^{1}-\mathrm{C}-\mathrm{H}\right), 55.97\left(\mathrm{Ar}^{1}-\mathrm{OCH}_{3}\right), 55.63\left(\mathrm{Ar}^{1}-\mathrm{OCH}_{3}\right), 45.59$ $\left(\mathrm{O}=\mathrm{C}-\mathrm{CH}_{2}\right), 78.29(>\mathrm{C}-\mathrm{H})$.

Compound 4h. It is having the molecular formula $\mathrm{C}_{18} \mathrm{H}_{16} \mathrm{O}_{4} \mathrm{~F}_{2}$ which showed molecular ion peak in positive mode $(\mathrm{M}+\mathrm{Na})^{+}$at 357.2 in LC-MS. So the molar mass of the compound $-4 \mathrm{~h}$ is $334 \mathrm{~g} / \mathrm{mol}$. Yield-55\%, M.P- $176-180^{\circ} \mathrm{C}$.

${ }^{1} \mathrm{HNMR}\left(400 \mathrm{MHz}, \delta\right.$ in ppm, $\left.\mathrm{CDCl}_{3}\right): 1 \mathrm{H}, \mathrm{t}, 7.34\left(\mathrm{Ar}^{1}-\mathrm{H}\right)$, $2 \mathrm{H}, \mathrm{m}, 7.2\left(\mathrm{Ar}^{1}-\mathrm{H}\right), 1 \mathrm{H}, \mathrm{s}, 6.1(\mathrm{Ar}-\mathrm{C}-\mathrm{H}), 1 \mathrm{H}, \mathrm{dd}, 5.3\left(>\mathrm{C}<_{\mathrm{H}}\right)$, $3 \mathrm{H}, \mathrm{s}, 3.9\left(\mathrm{Ar}-\mathrm{OCH}_{3}\right), 3 \mathrm{H}, \mathrm{s}, 3.9\left(\mathrm{Ar}-\mathrm{OCH}_{3}\right), 2 \mathrm{H}, \mathrm{m}, 2.8(\mathrm{O}=\mathrm{C}-$ $\left.\mathrm{CH}_{2}\right), 3 \mathrm{H}, \mathrm{s}, 2.05\left(\mathrm{Ar}-\mathrm{CH}_{3}\right)$.

${ }^{13} \mathrm{C}$ NMR: $\left(\mathrm{CDCl}_{3}, 100 \mathrm{MHz}, \delta\right.$ in $\left.\mathrm{ppm}\right) 7.81$ (Ar$\left.\mathrm{CH}_{3}\right), 106.32\left(\mathrm{Ar}-\mathrm{C}-\mathrm{CH}_{3}\right), 160.64\left(\mathrm{Ar}-\mathrm{C}-\mathrm{OCH}_{3}\right), 88.32(\mathrm{Ar}-$ $\mathrm{C}-\mathrm{H}), 163.71\left(\mathrm{Ar}-\mathrm{C}-\mathrm{OCH}_{3}\right), 105.85$ ( $\left.\mathrm{Ar}-\mathrm{C}-\mathrm{C}=\mathrm{O}\right), 160.59$ (Ar-C-O), $56.08\left(\mathrm{Ar}-\mathrm{OCH}_{3}\right), 55.66\left(\mathrm{Ar}-\mathrm{O}-\mathrm{CH}_{3}\right), 189.02$ $(\mathrm{Ar}-\mathrm{C}=\mathrm{O}), 136.44\left(\mathrm{Ar}^{1}-\mathrm{C}<\right), 115.21\left(\mathrm{Ar}^{1}-\mathrm{C}-\mathrm{H}\right), 121.13\left(\mathrm{Ar}^{1}-\right.$ C-F), 121.84 ( $\left.\mathrm{Ar}^{1}-\mathrm{C}-\mathrm{F}\right), 115.02\left(\mathrm{Ar}^{1}-\mathrm{C}-\mathrm{H}\right), 117.6\left(\mathrm{Ar}^{1}-\mathrm{C}-\mathrm{H}\right)$, $45.54\left(\mathrm{O}=\mathrm{C}-\mathrm{CH}_{2}\right), 77.32(>\mathrm{C}-\mathrm{H})$.
Compound 5. It is having the molecular formula $\mathrm{C}_{16} \mathrm{H}_{14} \mathrm{O}_{5}$ which showed molecular ion peak in negative mode $(\mathrm{M}-\mathrm{H})^{-}$at 285.1 in LC-MS. So the molar mass of the compound 5 is $286 \mathrm{~g} / \mathrm{mol}$.

${ }^{1} \mathrm{H}$ NMR: $\left(\mathrm{CDCl}_{3}, 400 \mathrm{MHz}, \delta\right.$ in ppm): $1 \mathrm{H}, \mathrm{s}, 9.65$ (Ar$\mathrm{OH}), 1 \mathrm{H}, \mathrm{s}, 9.407$ (Ar-OH), 1H, s 9.339 ( $\left.\mathrm{Ar}^{1}-\mathrm{OH}\right), 1 \mathrm{H}, \mathrm{t} 7.030$ $\left(\mathrm{Ar}^{1}-\mathrm{OH}\right), 1 \mathrm{H}, \mathrm{d} 6.822\left(\mathrm{Ar}^{1}-\mathrm{OH}\right), 1 \mathrm{H}, \mathrm{t} 6.622\left(\mathrm{Ar}^{1}-\mathrm{H}\right), 1 \mathrm{H}, \mathrm{t}$, $6.44\left(\mathrm{Ar}^{1}-\mathrm{H}\right), 1 \mathrm{H}, \mathrm{s} 6.260(\mathrm{Ar}-\mathrm{H}), 1 \mathrm{H}, \mathrm{d}, 4.636(\mathrm{O}-\mathrm{C}-\mathrm{H}), 2 \mathrm{H}$, dd $3.047\left(\mathrm{O}=\mathrm{C}-\mathrm{CH}_{2}\right), 3 \mathrm{H}, \mathrm{s}, 1.984\left(\mathrm{Ar}-\mathrm{CH}_{3}\right)$.

${ }^{13} \mathrm{C} \mathrm{NMR:}\left(\mathrm{CDCl}_{3}, 400 \mathrm{MHz}, \delta\right.$ in ppm) $7.81\left(\mathrm{Ar}-\mathrm{CH}_{3}\right)$, $106.36\left(\mathrm{Ar}-\mathrm{C}-\mathrm{CH}_{3}\right), 163.56$ (Ar-C-OH), 88.64 (Ar-C-H), 161.52 (Ar-C-OH), 105.98 ( $\mathrm{Ar}-\mathrm{C}-\mathrm{C}=\mathrm{O}), 160.52$ (Ar-C-O), $189.83(\mathrm{Ar}-\mathrm{C}=\mathrm{O}), 129.73\left(\mathrm{Ar}^{1}-\mathrm{C}<\right), \delta$ at $159.90\left(\mathrm{Ar}^{1}-\mathrm{C}-\right.$ $\mathrm{OH}), 113.63$ ( $\left.\mathrm{Ar}^{1}-\mathrm{C}-\mathrm{H}\right), 140.99\left(\mathrm{Ar}^{1}-\mathrm{C}-\mathrm{H}\right), 111.58\left(\mathrm{Ar}^{1}-\mathrm{C}-\right.$ $\mathrm{H}), 118.01\left(\mathrm{Ar}^{1}-\mathrm{C}-\mathrm{H}\right), 44.65\left(\mathrm{O}=\mathrm{C}-\mathrm{CH}_{2}\right), \delta$ at $74.23(>\mathrm{C}-\mathrm{H})$.

\subsection{Bioactivity Studies}

3.2.1. Antituberculosis. Compounds $\mathbf{3} \mathbf{a}-\mathbf{h}, \mathbf{4 a}-\mathbf{h}$, and $\mathbf{5}$ (pisonivanone) were screened for antituberculosis on Myco 
TABle 3: Antioxidant and cytotoxic activity of Flavanones (Compounds $\mathbf{4 a - h}$ ).

\begin{tabular}{|c|c|c|c|c|c|c|c|}
\hline \multirow{2}{*}{ Test name } & \multicolumn{3}{|c|}{ Superoxide inhibitory activity } & \multicolumn{3}{|c|}{ DPPH inhibitory activity } & \multirow{2}{*}{$\begin{array}{l}\text { Brine shrimp Lethality test } \\
\text { ED50 }\end{array}$} \\
\hline & Dose at $(\mu \mathrm{g} / \mathrm{mL})$ & $\%$ of inhibition & IC50 & Dose at $(\mu \mathrm{g} / \mathrm{mL})$ & $\%$ of inhibition & IC50 & \\
\hline \multirow{3}{*}{ Compound-4a } & 10 & 13.89 & \multirow{3}{*}{44.99} & 10 & 11.27 & \multirow{3}{*}{48.77} & \multirow{3}{*}{34.11} \\
\hline & 25 & 37.58 & & 25 & 35.76 & & \\
\hline & 50 & 54.41 & & 50 & 58.18 & & \\
\hline \multirow{3}{*}{ Compound-4b } & 10 & 26.58 & \multirow{3}{*}{29.18} & 10 & 14.06 & \multirow{3}{*}{36.35} & \multirow{3}{*}{22.55} \\
\hline & 25 & 48.67 & & 25 & 40.73 & & \\
\hline & 50 & 72.00 & & 50 & 65.21 & & \\
\hline \multirow{3}{*}{ Compound-4c } & 10 & 24.02 & \multirow{3}{*}{33.00} & 10 & 25.61 & \multirow{3}{*}{29.24} & \multirow{3}{*}{31.22} \\
\hline & 25 & 43.30 & & 25 & 42.68 & & \\
\hline & 50 & 67.65 & & 50 & 78.15 & & \\
\hline \multirow{3}{*}{ Compound-4d } & 10 & 20.75 & \multirow{3}{*}{32.68} & 10 & 26.20 & \multirow{3}{*}{25.81} & \multirow{3}{*}{27.33} \\
\hline & 25 & 47.55 & & 25 & 57.34 & & \\
\hline & 50 & 67.16 & & 50 & 77.29 & & \\
\hline \multirow{3}{*}{ Compound-4e } & 10 & 28.27 & \multirow{3}{*}{28.77} & 10 & 9.70 & \multirow{3}{*}{42.39} & \multirow{3}{*}{41.82} \\
\hline & 25 & 49.02 & & 25 & 37.94 & & \\
\hline & 50 & 71.41 & & 50 & 55.76 & & \\
\hline \multirow{3}{*}{ Compound-4f } & 10 & 10.23 & \multirow{3}{*}{39.03} & 10 & 18.30 & \multirow{3}{*}{38.08} & \multirow{3}{*}{52.15} \\
\hline & 25 & 32.81 & & 25 & 39.27 & & \\
\hline & 50 & 64.06 & & 50 & 61.45 & & \\
\hline \multirow{3}{*}{ Compound-4g } & 10 & 17.65 & & 10 & 17.82 & & \\
\hline & 25 & 42.16 & 36.24 & 25 & 46.79 & 32.28 & 28.93 \\
\hline & 50 & 63.73 & & 50 & 70.30 & & \\
\hline & 10 & 17.34 & & 10 & 16.48 & & \\
\hline Compound-4h & 25 & 41.35 & 31.95 & 25 & 43.64 & 33.28 & 42.56 \\
\hline & 50 & 75.64 & & 50 & 70.30 & & \\
\hline & 5 & 12.42 & & 10 & 31.53 & & \\
\hline Compound-5 & 10 & 46.41 & 25.32 & 25 & 64.50 & 20.14 & 14.35 \\
\hline & 25 & 66.18 & & 50 & 86.76 & & \\
\hline & 2.5 & 34.49 & & 2.5 & 34.49 & & \\
\hline Vit-C & 5 & 62.71 & 3.98 & 5 & 62.71 & 3.98 & Phodophyllotoxin 3.69 \\
\hline & 10 & 102.27 & & 10 & 102.27 & & \\
\hline
\end{tabular}

bacterium tuberculosis in LJ medium by using convention method [16] as follows.

Drug Free media (Lowenstein Jensen media) base was prepared according to manufacturer's instructions (HiMedia, Merck). The culture media was prepared by weighing 18.65 grams of the LJ medium base and dissolved in $300 \mathrm{~mL}$ of distilled water. Six $\mathrm{mL}$ glycerol (reagent grade) was added. The solution was autoclaved at $121^{\circ} \mathrm{C}$ for 30 minutes and cooled. $500 \mathrm{~mL}$ of homogenized whole egg was added and mixed. Then 6-8 $\mathrm{mL}$ of the medium was dispensed into a $28 \times$ $110 \mathrm{~mm}$ screw capped Mac Cartney culture tubes (Universal containers). These culture tubes were inspissated at $85^{\circ} \mathrm{C}$ for 55 minutes. For sterility check, prepared culture media were incubated at $37^{\circ} \mathrm{C}$ for 48 hours and were kept in the refrigerator for storage when no bacterial contaminants were detected. All tubes were tightly capped to prevent evaporation during storage.
Drug containing media was also prepared as above. Drug containing media was prepared as given in Table 1 . Stock solution was prepared by dissolving the $10 \mathrm{mg}$ of drug (Compounds 3a-h, 4a-h, and Compound 5) in $5 \mathrm{~mL}$ of DMF. Final concentration of stock solution is considered as $2000 \mathrm{mg} / \mathrm{L}$.

3.2.2. Drug Susceptibility Test. MTB isolate (preferably H37 $\mathrm{Rv)}$ were tested for drug susceptibility using the absolute concentration method on egg-based LJ medium, using a standardized inoculums $(4 \mathrm{mg} / 4 \mathrm{~mL})$ grown on drug free media along with media containing graded concentrations of the drug(s). All the test cultures are kept incubator at $37^{\circ} \mathrm{C}$ for 4-6 weeks.

The susceptibility test of antitubercular drugs was examined using five concentrations of Compounds $\mathbf{3 a}-\mathbf{h}, \mathbf{4 a}-\mathbf{h}$, and $\mathbf{5}(12.5,25,50,100$, and $200 \mathrm{mg} / \mathrm{L})$. 
Resistance is expressed in terms of the lowest concentration of the drug that inhibits growth, that is, minimal inhibitory concentration (MIC).

\subsection{Antioxidant Activity.}

Superoxide Scavenging Method. Superoxide scavenging activity of the compounds $\mathbf{3 a - h}, \mathbf{4 a - h}, \mathbf{5 a}-\mathbf{e}$, and $\mathbf{6 a - e}$ was determined by the method [17] modified by [18] Kutlan et al., which depends on the light induced superoxide generation by riboflavin and the corresponding reduction of NBT. The assay mixture contained different concentration of the test substances and EDTA ( $6 \mathrm{mM}$ containing $3 \mu \mathrm{g} \mathrm{NaCN})$, NBT $(50 \mu \mathrm{M})$ riboflavin $(2 \mu \mathrm{M})$, and phosphate buffer $58 \mathrm{mM}, \mathrm{pH}$ 7.8 in a total volume of $3 \mathrm{~mL}$. The tubes received uniform illumination for $15 \mathrm{~min}$ and thereafter optical density was measured at $560 \mathrm{~nm}$. Consider the following:

$$
\% \text { inhibition }=\left[\frac{(\text { control }- \text { sample })}{\text { control }}\right] \times 100 \text {. }
$$

An $\mathrm{IC}_{50}$ value was determined as the concentration that elicited the half maximal response.

Stastical Analysis. The data were analyzed by (single factor) for multiple groups and the significance level was chosen as $P<0.05$. Data is expressed as the mean $(+)$ or $(-)$-SEM with a minimum of three experiments performed per each variable.

3.4. DPPH Free Radical Scavenging Activity. DPPH $(1,1-$ diphenyl-2picryl-hydrazyl) free radical scavenging activity of the synthesised compounds $\mathbf{3 a}-\mathbf{h}, \mathbf{4 a}-\mathbf{h}$, and $\mathbf{5}$ was determined by the method [19] of Lamaison et al., which depends on scavenging of colored free radical (DPPH) in methanol solution by the test drugs. The reaction mixture contains DPPH and test drug in a final concentration of $3 \mathrm{~mL}$. Absorption of DPPH at its adsorption maximum $516 \mathrm{~nm}$ is inversely proportional to the concentration of the scavenger (test drug). The activity was expressed as inhibitory concentration $50\left(\mathrm{IC}_{50}\right)$, that is, the concentration of the test solution required to give $50 \%$ reduction in absorbance of the test solution as compared to that of blank solution. Consider

$$
\begin{aligned}
\mathrm{IC}_{50}= & {\left[\frac{(\mathrm{OD} \text { of control }-(\mathrm{OD} \text { of test }-\mathrm{OD} \text { test blank }))}{\mathrm{OD} \text { of control }}\right] } \\
& \times 100 .
\end{aligned}
$$

The results obtained by both methods were given in Tables 2 and 3.

3.5. Cytotoxic Activity. Cytotoxic activity of the synthesised compounds $\mathbf{3} \mathbf{a}-\mathbf{h}, \mathbf{4 a}-\mathbf{h}$, and $\mathbf{5}$ were determined by using a known method $[20,21] .5 \mathrm{~mL}$ of brine solution is taken into each test tube. Test substances were added to the tube according to their concentrations. The solutions were thoroughly mixed with the help of the cyclomixer. Then 10 shrimps were added to each test tube. Suitable conditions like temperature should be maintained for proper results. Finally by observing the lethality rate of the shrimps after 24 hours the $\mathrm{ED}_{50}$ value is calculated. The $\mathrm{ED}_{50}$ using probed analysis at $95 \%$ confidence limits from observed data. Replicas maintained to get accurate results (Tables 2 and 3 ).

\section{Acknowledgments}

The authors are thankful to Acharya Nagarjuna University, Guntur, A.P-India, and UGC, New Delhi (UGC-MRP/F. no. 39-752/2010 (SR) dated 13.01.2011) for their financial support. Also they are greatly thankful to G. V. Subba Raju, Director and C.E.O. Natsol Laboratories Pvt. Ltd., for his helpful discussions.

\section{References}

[1] L. H. Yao, Y. M. Jiang, J. Shi et al., "Flavonoids in food and their health benefits," Plant Foods for Human Nutrition, vol. 59, no. 3, pp. 113-122, 2004.

[2] K. Likhitwitayawuid, T. Phadungcharoen, and J. Krungkrai, "Antimalarial xanthones from Garcinia cowa," Planta Medica, vol. 64, no. 1, pp. 70-72, 1998.

[3] H. Haraguchi, H. Ishikawa, K. Mizutani, Y. Tamura, and T. Kinoshita, "Antioxidative and superoxide scavenging activities of retrochalcones in Glycyrrhiza inflata," Bioorganic and Medicinal Chemistry, vol. 6, no. 3, pp. 339-347, 1998.

[4] Y. Satomi, "Inhibitory effects of 3'-methyl-3-hydroxy-chalcone on proliferation of human malignant tumor cells and on skin carcinogenesis," International Journal of Cancer, vol. 55, no. 3, pp. 506-514, 1993.

[5] Y. B. Ryu, M. J. Curtis-Long, J. W. Lee et al., "Structural characteristics of flavanones and flavones from Cudrania tricuspidata for neuraminidase inhibition," Bioorganic and Medicinal Chemistry Letters, vol. 19, no. 17, pp. 4912-4915, 2009.

[6] R. J. Anto, K. Sukumaran, G. Kuttan, M. N. A. Rao, V. Subbaraju, and R. Kuttan, "Anticancer and antioxidant activity of synthetic chalcones and related compounds," Cancer Letters, vol. 97, no. 1, pp. 33-37, 1995.

[7] J. F. Ballesteros, M. J. Sanz, A. Ubeda et al., "Synthesis and pharmacological evaluation of $2^{\prime}$-hydroxychalcones and flavones as inhibitors of inflammatory mediators generation," Journal of Medicinal Chemistry, vol. 38, no. 14, pp. 2794-2797, 1995.

[8] B. P. Bandgar and S. S. Gawande, "Synthesis and biological screening of a combinatorial library of $\beta$-chlorovinyl chalcones as anticancer, anti-inflammatory and antimicrobial agents," Bioorganic and Medicinal Chemistry, vol. 18, no. 5, pp. 2060-2065, 2010.

[9] M. C. Wu, C. F. Peng, I. S. Chen, and I. L. Tsai, "Antitubercular chromones and flavonoids from Pisonia aculeata," Journal of Natural Products, vol. 74, no. 5, pp. 976-982, 2011.

[10] F. H. Curd and A. Robertson, "114. Usnic acid. Part I. Derivatives of methylphloroglucinol," Journal of the Chemical Society, pp. 437-444, 1933.

[11] K. Aghoramurthy, N. Narascimhachari, and T. R. Seshadri, "Synthetic experiments in the benzopyrone series. Part XVI. Isoformononetin and its derivatives," Proceedings of the Indian Academy of Sciences A, vol. 33, pp. 257-263, 1951.

[12] V. K. Ahluwalia, P. Bhagat, R. Agarwal, and R. Chandra, Intermadiates for Organic Synthesis, I.K International Pvt.Ltd., 2005. 
[13] Y. Rajendra Prasad, A. Lakshmana Rao, and R. Rambabu, "Synthesis and antimicrobial activity of some chalcone derivatives," E-Journal of Chemistry, vol. 5, no. 3, pp. 461-466, 2008.

[14] T. Narender and K. Papi Reddy, "A simple and highly efficient method for the synthesis of chalcones by using borontrifluoride-etherate," Tetrahedron Letters, vol. 48, no. 18, pp. 3177-3180, 2007.

[15] S. Eddarir, N. Cotelle, Y. Bakkour, and C. Rolando, "An efficient synthesis of chalcones based on the Suzuki reaction," Tetrahedron Letters, vol. 44, no. 28, pp. 5359-5363, 2003.

[16] Standard Operating Procedures (SOPs) for Tuberculosis Lab, Procedure no. 03. 01., developed by central TB Division, Directorate General of Health Services, Ministry of health and Family Welfare, New Delhi, India.

[17] J. M. McCord and I. Fridovich, "Superoxide dismutase. An enzymic function for erythrocuprein (hemocuprein)," The Journal of Biological Chemistry, vol. 244, no. 22, pp. 6049-6055, 1969.

[18] R. Kutlan, A. John, G. kutlan, K. V. Dinesh Babu, and K. N. Raja sekarau, "Antitumor and free radicals scavenging activity of synthetic cucuminoids," International Journal of Pharmaceuticals, vol. 131, 1996.

[19] J. L. Lamaison, C. Petitjean-Freytet, and A. Carnat, "Medicinal lamiaceae with antioxidative activities, potential sources of rosmarinic acid," Pharmaceutica Acta Helvetiae, vol. 66, no. 7, pp. 185-188, 1991.

[20] B. N. Meyer, N. R. Ferrigni, and J. E. Putnam, "Brine shrimp: a convenient general bioassay for active plant constituents," Planta Medica, vol. 45, no. 1, pp. 31-34, 1982.

[21] A. V. Krishnaraju, T. V. N. Rao, D. Sundararaju, M. Vanisree, H. S. Tsay, and G. V. Subbaraju, "Biological screening of medicinal plants collected from Eastern Ghats of India using Artemia salina (brine shrimp test)," International Journal of Applied Science and Engineering, vol. 4, no. 2, pp. 115-125, 2006. 

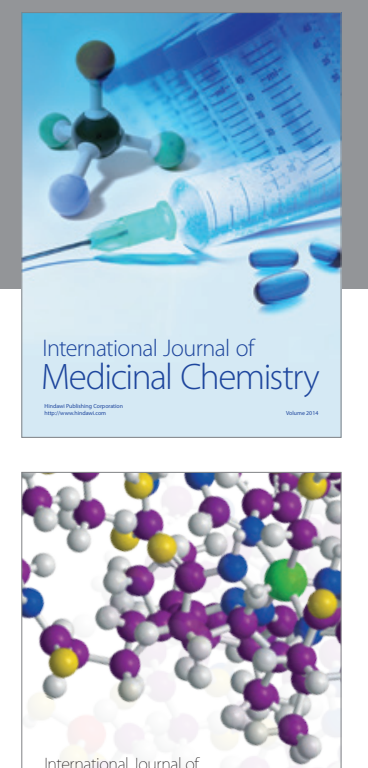

\section{Carbohydrate} Chemistry

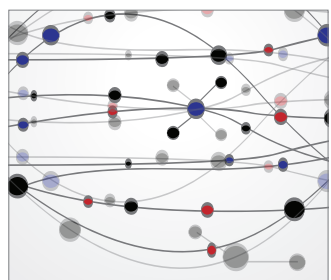

The Scientific World Journal
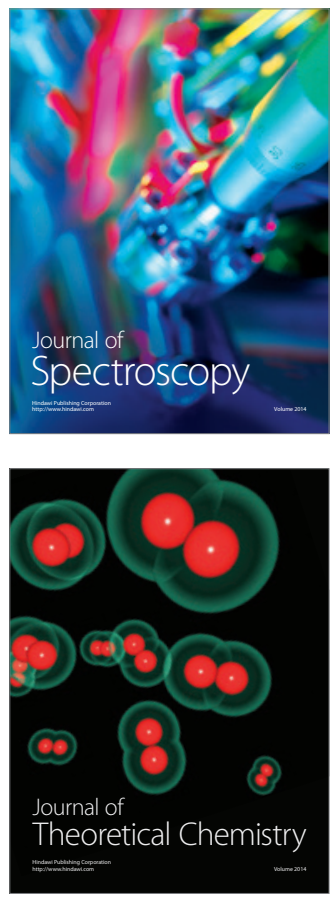
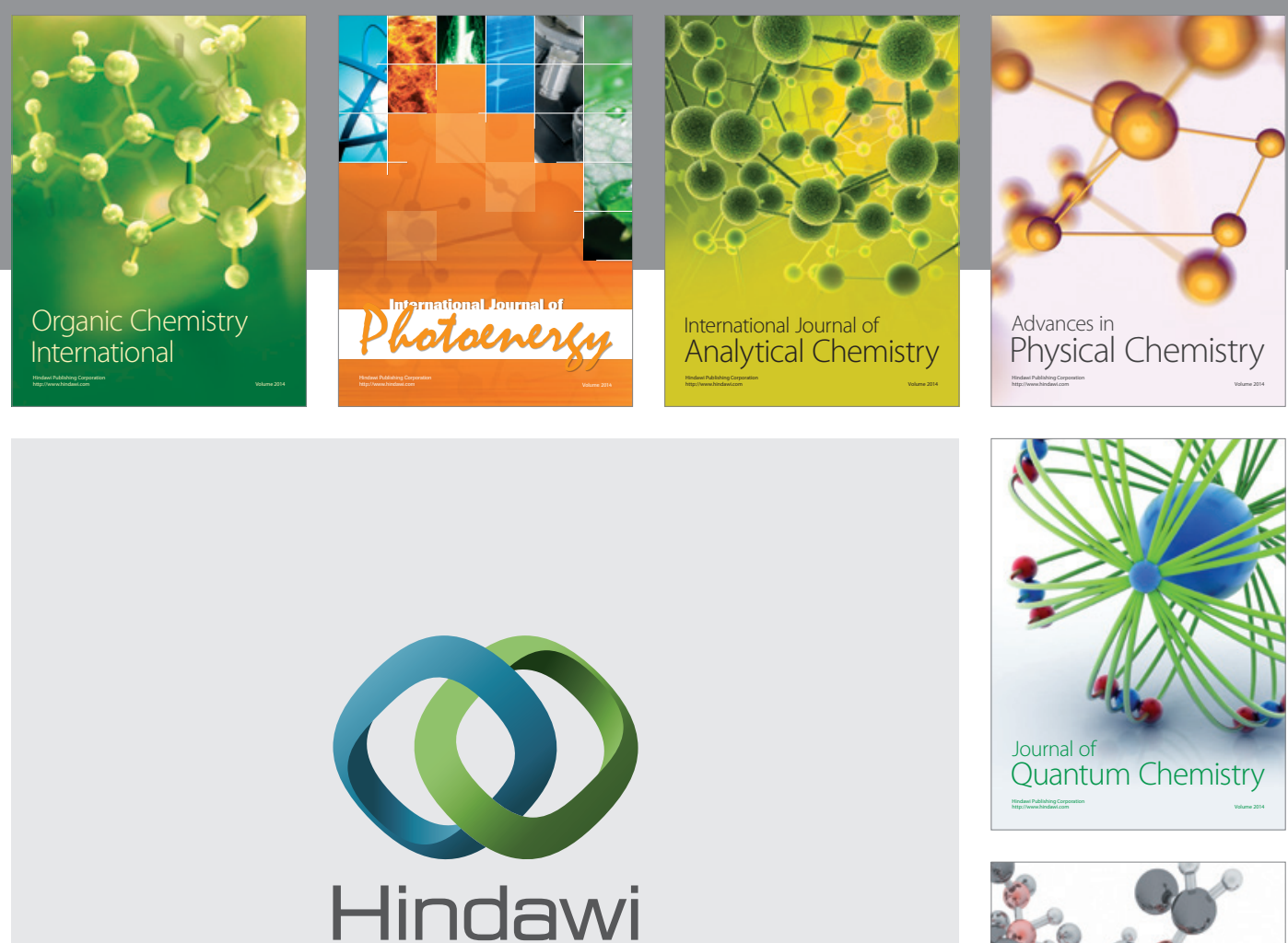

Submit your manuscripts at

http://www.hindawi.com

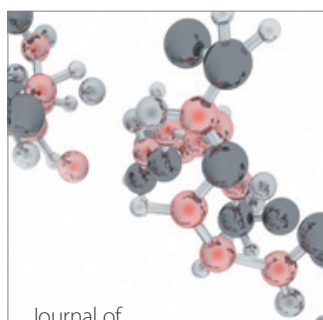

Analytical Methods

in Chemistry

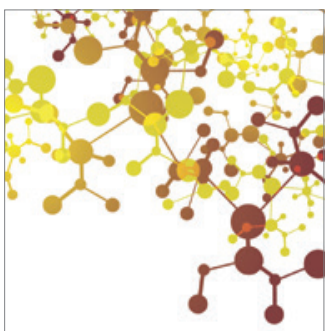

Journal of

Applied Chemistry

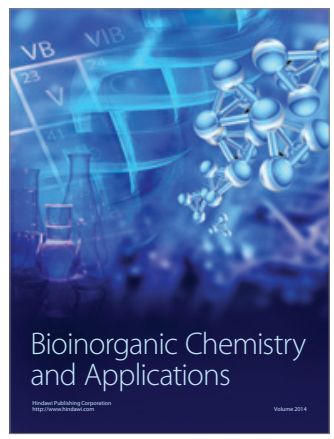

Inorganic Chemistry
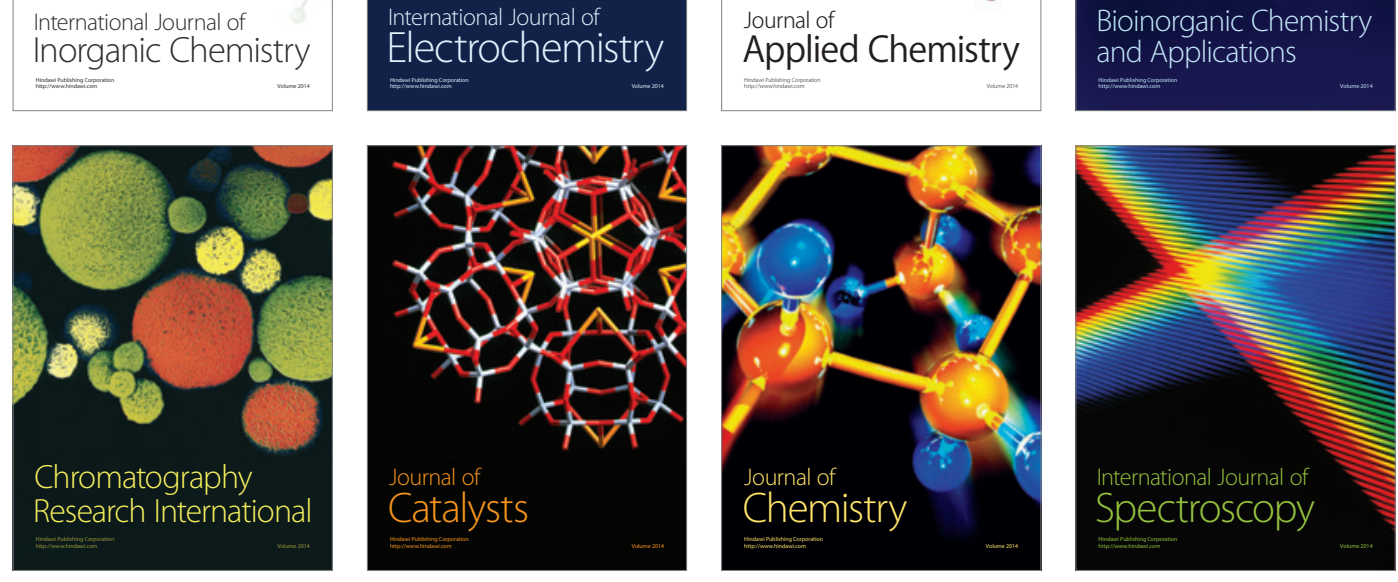